\title{
Epidemiological Pattern of Traumatic Brain Injury in Brazil between 2008 and 2019
}

\section{Padrão epidemiológico de traumatismo cranioencefálico no Brasil entre 2008 e 2019}

\author{
André Akira Ramos Takahashi ${ }^{1}$ Saulo Barros Teixeira ${ }^{2(0)}$ Giovanna Zambo Galafassi ${ }^{10}$ \\ Maria Beatriz Almeida Silva ${ }^{10}$ Victoria Fernandez Comprido ${ }^{10} \quad$ Anne Gabriele Senne Martinez ${ }^{10}$ \\ Leonardo Alfano de Lima ${ }^{30}$ Luiz Vinicius de Alcântara Sousa ${ }^{3}$ Paulo Henrique Pires de Aguiar ${ }^{4}$
}

\footnotetext{
${ }^{1}$ Faculdade de Medicina do ABC, Santo André, SP, Brazil

2 Department of Neurosurgery, Faculdade de Medicina do ABC, Santo

André, SP, Brazil

${ }^{3}$ Department of Epidemiology, Faculdade de Medicina do ABC, Santo

André, SP, Brazil

${ }^{4}$ Department of Neurology and Neurosurgery, Faculdade de Medicina do ABC, Santo André, SP, Brazil
}

\author{
Address for correspondence André Akira Ramos Takahashi, Av. Lauro \\ Gomes, 2000, Santo André, SP, Brazil \\ (e-mail: andreakiratakahashi@gmail.com).
}

Arq Bras Neurocir 2021;40(4):e303-e332.

\begin{abstract}
Introduction Traumatic brain injuries (TBIs) are a public health problem with high economic impact, as well as an important cause of death and sequela in polytrauma patients, affecting mainly young adults.

Objective To analyze the temporal trend of TBI incidence in Brazil between 2008 and 2019, according to age group and gender.

Methods An ecological study, based on secondary data from hospital admissions for TBI in all Brazilian states between 2008 and 2019. The numbers were collected using the hospital information system of the Unified Health System in Brazil. We performed a descriptive analysis using the data obtained. Linear regression models were used to measure the incidence trend of TBI in the period adopted.

Results The state of Piauí had the highest increase in the incidence of TBI in the country in the last 10 years (coefficient $\beta=63.43$ e $p=0.002$ ). The main concern, though, is the increase in the incidence of TBI amongst children ( $0-4$ years old) in the states of Ceará ( $\beta=31.22$ and $p<0.001$ for boys; $\beta=42.20$ and $p<0.001$ for girls), Paraná ( $\beta=37.26$ and $p=0.011$ for boys; $\beta=25.90$ and $p=0.015$ for girls), Pernam-

Keywords

- traumatic brain injury

- trauma

- incidence

- epidemiology

- Brazil buco $(\beta=20.08$ and $p=0.016$ for girls), Mato Grosso $(\beta=18.76$ and $p=0.005$ for boys; $\beta=16.11$ and $p=0.035$ for girls), and Distrito Federal $(\beta=48.87$ and $p=0.004$ for girls; $\beta=48.28$ and $p=0.006$ for boys).

Conclusion The analysis of the results is able to point out improvements that can be made. Besides that, it is remarkably important to redirect public polices to preventive medicine since many of the TBI causes are avoidable through awareness and education of the population.
\end{abstract}

received

September 1, 2020

accepted

March 9, 2021

published online

August 11, 2021
DOI https://doi.org/ 10.1055/s-0041-1733865. ISSN 0103-5355. (c) 2021. Sociedade Brasileira de Neurocirurgia. All rights reserved. This is an open access article published by Thieme under the terms of the Creative Commons Attribution-NonDerivative-NonCommercial-License, permitting copying and reproduction so long as the original work is given appropriate credit. Contents may not be used for commercial purposes, or adapted, remixed, transformed or built upon. (https://creativecommons.org/ licenses/by-nc-nd/4.0/)

Thieme Revinter Publicações Ltda., Rua do Matoso 170, Rio de Janeiro, RJ, CEP 20270-135, Brazil 


\section{Resumo}

\section{Palavras-chave \\ - traumatismo cranioencefálico \\ - trauma \\ - incidência \\ - epidemiologia \\ - Brasil}

Introdução O traumatismo cranioencefálico (TCE) é um problema de saúde pública com alto impacto econômico, além de uma importante causa de morte e sequela em pacientes politraumatizados, afetando principalmente adultos jovens.

Objetivo Analisar a tendência temporal da incidência de TCE no Brasil entre 2008 e 2019, segundo faixa etária e sexo.

Método Estudo ecológico, baseado em dados secundários de internações hospitalares por TCE em todos os estados brasileiros entre 2008 e 2019. Os números foram coletados por meio do sistema de informações hospitalares do Sistema Único de Saúde do Brasil. Realizamos uma análise descritiva a partir dos dados obtidos. Modelos de regressão linear foram utilizados para medir a tendência da incidência de TCE no período adotado.

Resultados O estado do Piauí apresentou o maior aumento na incidência de TCE no país nos últimos dez anos (coeficiente $\beta=63,43$ e $p=0,002$ ). A principal preocupação, porém, é o aumento da incidência de TCE entre crianças (0-4 anos) nos estados do Ceará ( $\beta=31,22$ e $p<0,001$ para meninos; $\beta=42,20$ e $p<0,001$ para meninas), Paraná ( $\beta=37,26$ e $p=0,011$ para meninos; $\beta=25,90$ e $p=0,015$ para meninas), Pernambuco ( $\beta=20,08$ e $p=0,016$ para meninas), Mato Grosso $(\beta=18,76$ e $p=0,005$ para meninos; $\beta=16,11$ e $p=0,035$ para meninas) e Distrito Federal ( $\beta=48,87$ e $p=0,004$ para meninas; $\beta=48,28$ e $p=0,006$ para meninos).

Conclusão $A$ análise dos resultados é capaz de apontar melhorias que podem ser feitas. Além disso, é extremamente importante redirecionar as políticas públicas para a medicina preventiva, uma vez que muitas das causas do TCE são evitáveis por meio da conscientização e educação da população.

\section{Introduction}

Traumatic brain injuries (TBIs) are a public health problem with high economic impact. They are an important cause of deaths and sequela in polytrauma patients, ${ }^{1}$ affecting mainly young adults. ${ }^{2,3}$ The World Health Organization (WHO) estimates that TBIs are responsible for one third to half of trauma deaths worldwide. ${ }^{1}$ Their incidence changes according to country and region, and there is an estimation that almost $90 \%$ of deaths from injuries occur in countries with low and middle incomes. ${ }^{1}$ Moreira Jr et al. mentioned that car accidents, being run over, cycling and motorcycle accidents, physical aggressions, falls, and firearm injuries are the main causes of TBI. ${ }^{4}$

The actual incidence of TBI changes according to place due to underdiagnosis, lower assistance to patients, and lack of monitoring and registration systems in many health care units. $^{5-7}$ The incidence of TBI is approximately $538.2 / 1,000.00$ inhabitants in the USA, ${ }^{1} 322 / 1,000.00$ in Australia, ${ }^{1}$ and $235 / 1,000.00$ in the European Union, ${ }^{1}$ with a remarkable variability in the last one. Germany had an incidence of 340/1,000.00 inhabitants ${ }^{2}$; Finland, 101/1,000.00, ${ }^{2}$ and Italy, $212-372 / 1,000.00 .^{2}$ The mortality rate also varies worldwide from 15 to 24.6/1,000.00.2,8,9 In Brazil, the Southeast (42.5\%) and Northeast (25.8\%) areas of the country have the highest incidence of cases in the country. ${ }^{1,10}$

TBI is an anatomical and/or functional lesion that affects brain, meninges, neurovascular components, skull and even scalp, ${ }^{1}$ occurring due to physical impact. ${ }^{1,2}$ Traumatic brain injury can cause temporary or permanent sequela in cognitive, behavioral, emotional and physical performances. The clinical features of a TBI patient are represented by conscious changes; neuromuscular function, sensory, language, personality and visual disorders; epilepsy; incontinence; cranial nerve palsy; changes in the autonomic function and abnormal postures, which may be the signs of decortication or decerebration. ${ }^{4,11}$ TBI classification depends on the severity (mild, moderate or severe), morphology (extra or intracranial injuries) and on the mechanism of trauma (blunt or penetration). ${ }^{1}$

The diagnosis of TBI involves an extensive neurological examination. The Glasgow coma scale is used to evaluate the severity of the trauma. ${ }^{3}$ The interventions may vary between postural measures, pharmacological therapies, monitoring, induced coma and even craniotomy. ${ }^{3}$

In Brazil and in other Latin American countries, little is known about this topic. In Brazil, there is an estimative of 100,000 deaths per year, furthermore over one million people became disabled between the years of 2007 and $2017,{ }^{1}$ which has a massive impact in the countrýs socioeconomics.

Considering this scenario, this study aims to analyze the temporal trend of TBI incidence in Brazilian states between 2008 and 2019, according to age group and gender. This research aims to help the development of preventive measures and hospital interventions that may reduce TBI incidence and its sequelaes. ${ }^{12}$ 


\section{Methods}

This ecological study was conducted based on secondary data from hospital admissions for TBI in all Brazilian states between 2008 and 2019. Data was collected from the Department of Informatics of the Unified Health System (DATASUS). The Department of Informatics of the Unified Health System is the official database of the Brazilian Health Ministry and can be visited for free on the website www.datasus.gov.br.

We searched DATASUS for the number of hospital admissions between 2008 and 2019, including female/male patients in different age groups, from 0 to 80 years old, all identified by the ICD 10 -S09. To collect these data, the demographic census from 2010 and the linter-census projections (2011-2019) of the Brazilian Institute of Geography and Statistics (IBGE, in the Portuguese acronym) were used. This demographic information can also be found in the DATASUS system.

The incidence rates of TBI were calculated using the formula: number of hospital admissions divided by the total population per 1,000.00 inhabitants, considering age groups, Brazilian states, gender, and years adopted in this study. Since we calculate the incidence of TBI in the general population stratified by the variables above, the population at risk of TBI was the total one of that state, in that year, age, and gender. The formula used is as follow:

Furthermore, hospital admission rates were used in a standard form and fitted to the age groups according to the direct method proposed by The World Health Organization (WHO), according to Ahmad et al. ${ }^{13}$

\section{Ethics Committee}

The present survey used secondary data available with unrestricted access on the DATASUS database. For this reason, there was no need to register this research with the Human Research Ethics Committee, according to the stipulated resolution 510/2016 of the Brazilian Health Ministry.

\section{Statistical Analysis}

We performed a descriptive analysis using the data obtained. Linear regression models were used to measure the incidence trend of TBI in the period adopted. This trend was estimated according to national standard rates by state and age group, with a 95\% confidence interval, using the Stata software version 11.0 (StataCorp., College Station, TX, USA).

\section{Results}

With the information obtained from DATASUS and IBGE, we calculated the incidence rates of hospital admissions due to TBI in Brazil from 2008 to 2019. The obtained data may be found in - Table 1 and - Table 2 . The results found in each region of the country, outlining the main data regarding each state, are described below.

\section{The North Region (Acre, Amapá, Amazonas, Pará, Rondônia, Roraima, Tocantins)}

In Acre, there was a statistically significant reduction in the incidence of hospital admissions for both female and male patients ( $\beta$ coefficient: -2.09 e -0.86 , respectively). The largest reduction was observed in men in the 30 to 40 years old age group ( $\beta$ coefficient: -51.15).

In Amazonas, the total incidence of admissions increased in the female gender ( $\beta$ coefficient: 0.66), mainly in the age extremes ( $0-4$ and $\geq 75$ years old). In the male gender, the global increase of the incidence of TBI in 2.5 times ( $\beta$ coefficient: 2.53) was a result of the higher number of cases in practically all groups, with the exception of the age groups 5 to 9,10 to 14 , and 35 to 39 years old.

In Amapá, there was a higher reduction in male ( $\beta$ coefficient: -5.35 ), when compared to female ( $\beta$ coefficient - 2.54). In Pará, there was an increase in the incidence in both genders, though more significant in the male gender $(\beta$ coefficient 3.05).

In Roraima, males $\geq 80$ years old were the only individuals that obtained a statistically significance result, with an expressive increase in the hospital admissions of approximately 31 times ( $\beta$ coefficient: 31.04). Similarly, in Rondônia, men $\geq 65$ years old presented an increase in admissions ( $\beta$ coefficient: 21.17-26.12).

In Tocantins, men in the 25 to 29 ( $\beta$ coefficient: -40.35 ), 30 to 34 ( $\beta$ coefficient: -26.68 ) and 60 to 64 years old ( $\beta$ coefficient: - 16.98) age groups presented an important reduction in hospital admissions for severe TBI.

\section{The Northeast Region (Alagoas, Bahia, Ceará, Maranhão, Paraíba, Pernambuco, Piauí, Rio Grande do Norte, and Sergipe)}

Alagoas showed an increase in the incidence of admissions for TBI, mainly among seniors $\geq 80$ years old, of both genders; however, the increase was higher in males ( $\beta$ coefficient: 7.01 and 2.91, males and females, respectively).

In Bahia, it was noticed an increase in the incidence of TBI in men of all age ranges > 50 years old, being more expressive in those 55 to 59 years old ( $\beta$ coefficient: 7.34). In women, the increase was also restricted to the older groups (50-54, 6569 , and $\geq 75$ years old).

The group in the pediatric age range from 0 to 4 years old had a significant increase in the rate of admissions for TBI in Ceará, presenting a rise from 30 to 40 times ( $\beta$ coefficient : 31.22 and 42.20 , girls and boys respectively). Curiously, there was a reduction among the boys from 10 to 14 years old ( $\beta$ coefficient: - 8.32). Among men, practically all age ranges from 40 years old on presented an increase in the incidence of TBI ( $\beta$ coefficient : 2.42). In women, the incidence increased in the group $\geq 70$ years old.

In Maranhão, all age ranges in both genders presented an increase in the admissions, with the highest value found among men in the 20 to 24 age group ( $\beta$ coefficient : 56.40 ). Comparing men and women, the increase was more expressive in men (approximately five times against once and a half in women). 
Table 1 Analysis of the age-adjusted incidence of TBI in the male population in each state of Brazil (2008-2019)

\begin{tabular}{|c|c|c|c|c|}
\hline Age group (years old) & R squad** & $P^{*}$ & $\beta$ & Cl 95 \\
\hline \multicolumn{5}{|l|}{ Acre } \\
\hline $0-4$ & 0.0795 & 0.375 & 5.10547 & $(-7.136651 ; 17.34759)$ \\
\hline $5-9$ & 0.3554 & 0.041 & -9.542892 & $(-18.59855 ;-0.4872313)$ \\
\hline $10-14$ & 0.2099 & 0.134 & -7.208869 & $(-17.06214 ; 2.644406)$ \\
\hline $15-19$ & 0.1077 & 0.298 & -9.344817 & $(-28.29793 ; 9.608292)$ \\
\hline $20-24$ & 0.7267 & $<0.001$ & -39.35719 & $(-56.36322 ;-22.35116)$ \\
\hline $25-29$ & 0.1212 & 0.267 & -13.43727 & $(-38.93176 ; 12.05723)$ \\
\hline $30-34$ & 0.6173 & 0.002 & -51.1543 & $(-79.53651 ;-22.77209)$ \\
\hline $35-39$ & 0.0034 & 0.857 & -2.807886 & $(-36.72276 ; 31.10699)$ \\
\hline $40-44$ & 0.0132 & 0.722 & -5.012117 & $(-35.56998 ; 25.54575)$ \\
\hline $45-49$ & 0.4549 & 0.016 & -25.22307 & $(-44.67879 ;-5.767353)$ \\
\hline $50-54$ & 0.1564 & 0.203 & -18.45109 & $(-48.63944 ; 11.73726)$ \\
\hline $55-59$ & 0.0096 & 0.761 & -1.843246 & $(-15.00416 ; 11.31767)$ \\
\hline $60-64$ & 0.0401 & 0.533 & -9.045007 & $(-40.23392 ; 22.14391)$ \\
\hline $65-69$ & 0.3056 & 0.062 & -29.3718 & $(-60.57087 ; 1.827269)$ \\
\hline $70-74$ & 0.1480 & 0.217 & 11.9816 & $(-8.273395 ; 32.23659)$ \\
\hline $75-79$ & 0.0867 & 0.353 & -9.318702 & $(-30.62933 ; 11.99193)$ \\
\hline$\geq 80$ & 0.0329 & 0.573 & 4.783482 & $(-13.49278 ; 23.05975)$ \\
\hline Total & 0.3835 & 0.032 & -2.092477 & $(-3.961977 ;-0.2229762)$ \\
\hline \multicolumn{5}{|l|}{ Alagoas } \\
\hline $0-4$ & 0.0881 & 0.349 & 2.979944 & $(-3.775536 ; 9.735423)$ \\
\hline $5-9$ & 0.0671 & 0.416 & -1.890463 & $(-6.858497 ; 3.07757)$ \\
\hline $10-14$ & 0.0013 & 0.910 & 0.3706837 & $(-6.760287 ; 7.501654)$ \\
\hline $15-19$ & 0.2874 & 0.072 & 20.96181 & $(-2.296971 ; 44.2206)$ \\
\hline $20-24$ & 0.2738 & 0.081 & 22.5201 & $(-3.318983 ; 48.35919)$ \\
\hline $25-29$ & 0.1167 & 0.277 & 14.76384 & $(-13.85724 ; 43.38491)$ \\
\hline $30-34$ & 0.2225 & 0.122 & 14.51405 & $(-4.604401 ; 33.63251)$ \\
\hline $35-39$ & 0.0830 & 0.364 & 9.19833 & $(-12.34906 ; 30.74571)$ \\
\hline $40-44$ & 0.2874 & 0.072 & 9.412445 & $(-1.030397 ; 19.85529)$ \\
\hline $45-49$ & 0.6947 & 0.001 & 13.54793 & $(7.219659 ; 19.8762)$ \\
\hline $50-54$ & 0.3221 & 0.054 & 13.23871 & $(-0.292886 ; 26.7703)$ \\
\hline 55-59 & 0.1332 & 0.243 & 5.881362 & $(-4.688915 ; 16.45164)$ \\
\hline $60-64$ & 0.1376 & 0.235 & 5.211926 & $(-3.981128 ; 14.40498)$ \\
\hline $65-69$ & 0.1879 & 0.159 & 5.824383 & $(-2.706355 ; 14.35512)$ \\
\hline $70-74$ & 0.3011 & 0.065 & 8.920001 & $(-0.655728 ; 18.49573)$ \\
\hline $75-79$ & 0.2838 & 0.075 & 7.407483 & $(-0.8836607 ; 15.69863)$ \\
\hline$\geq 80$ & 0.3534 & 0.042 & 7.015065 & $(0.3284547 ; 13.70167)$ \\
\hline Total & 0.2778 & 0.078 & 1.598776 & $(-0.2174201 ; 3.414972)$ \\
\hline \multicolumn{5}{|l|}{ Amapá } \\
\hline $0-4$ & 0.0108 & 0.748 & -1.047774 & $(-8.117716 ; 6.022168)$ \\
\hline $5-9$ & 0.1249 & 0.260 & -6.154093 & $(-17.63206 ; 5.323877)$ \\
\hline $10-14$ & 0.1455 & 0.221 & -6.406707 & $(-17.34478 ; 4.531366)$ \\
\hline $15-19$ & 0.4625 & 0.015 & -34.86841 & $(-61.35306 ;-8.383762)$ \\
\hline
\end{tabular}


Table 1 (Continued)

\begin{tabular}{|c|c|c|c|c|}
\hline Age group (years old) & R squad ${ }^{* *}$ & $P^{*}$ & $\beta$ & $\mathrm{Cl} 95$ \\
\hline $20-24$ & 0.6243 & 0.002 & -66.53081 & $(-102.8953 ;-30.16635)$ \\
\hline $25-29$ & 0.6369 & 0.002 & -65.25679 & $(-99.97742 ;-30.53616)$ \\
\hline $30-34$ & 0.3497 & 0.043 & -35.47139 & $(-69.55122 ;-1.391551)$ \\
\hline $35-39$ & 0.2278 & 0.117 & -33.24859 & $(-76.38113 ; 9.883956)$ \\
\hline $40-44$ & 0.1837 & 0.164 & -25.44084 & $(-63.22208 ; 12.34041)$ \\
\hline $45-49$ & 0.6592 & 0.001 & -59.22816 & $(-89.2335 ;-29.22282)$ \\
\hline $50-54$ & 0.5130 & 0.009 & -40.15096 & $(-67.71369 ;-12.58823)$ \\
\hline $55-59$ & 0.3221 & 0.054 & -40.47169 & $(-81.83799 ; 0.8945964)$ \\
\hline $60-64$ & 0.0405 & 0.531 & -6.782712 & $(-30.05815 ; 16.49273)$ \\
\hline $65-69$ & 0.3782 & 0.033 & -48.56186 & $(-92.43133 ;-4.692402)$ \\
\hline $70-74$ & 0.3702 & 0.036 & -24.12211 & $(-46.29181 ;-1.952409)$ \\
\hline $75-79$ & 0.2632 & 0.088 & -33.21057 & $(-72.36543 ; 5.944293)$ \\
\hline$\geq 80$ & 0.1616 & 0.195 & -8.176361 & $(-21.2969 ; 4.944174)$ \\
\hline Total & 0.5351 & 0.007 & -5.351298 & $(-8.86571 ;-1.836887)$ \\
\hline \multicolumn{5}{|l|}{ Amazonas } \\
\hline $0-4$ & 0.6825 & 0.001 & 11.65 & ( 6.052592; 17.2515) \\
\hline $5-9$ & 0.1506 & 0.213 & 3.97 & $(-2.673814 ; 10.614)$ \\
\hline $10-14$ & 0.2290 & 0.116 & 3.097 & $(-.965813 ; 7.100647)$ \\
\hline $15-19$ & 0.4573 & 0.016 & 17.588 & $(4.086702 ; 31.08998)$ \\
\hline $20-24$ & 0.6572 & 0.001 & 19.802 & $(9.725824 ; 29.87979)$ \\
\hline $25-29$ & 0.6295 & 0.002 & 23.886 & $(10.97499 ; 36.79889)$ \\
\hline $30-34$ & 0.5296 & 0.007 & 18.261 & $(6.133773 ; 30.38902)$ \\
\hline $35-39$ & 0.2160 & 0.128 & 9.667 & $(-3.310196 ; 22.64535)$ \\
\hline $40-44$ & 0.4661 & 0.014 & 15.111 & $(3.716433 ; 26.50563)$ \\
\hline $45-49$ & 0.3693 & 0.036 & 9.819 & $(0.7768351 ; 18.86172)$ \\
\hline $50-54$ & 0.3349 & 0.049 & 11.417 & (0.0799973; 22.75599) \\
\hline $55-59$ & 0.7250 & $<0.001$ & 21.117 & $(11.95378 ; 30.282)$ \\
\hline $60-64$ & 0.4952 & 0.011 & 18.394 & $(5.307926 ; 31.4802)$ \\
\hline $65-69$ & 0.5703 & 0.005 & 20.945 & $(8.134567 ; 33.75685)$ \\
\hline $70-74$ & 0.5636 & 0.005 & 18.730 & $(7.117275 ; 30.34407)$ \\
\hline $75-79$ & 0.3802 & 0.033 & 15.767 & $(1.584023 ; 29.95156)$ \\
\hline$\geq 80$ & 0.5274 & 0.007 & 14.128 & $(4.705697 ; 23.55171)$ \\
\hline Total & 0.6972 & 0.001 & 2.533 & $(1.357071 ; 3.710116)$ \\
\hline \multicolumn{5}{|l|}{ Bahia } \\
\hline $0-4$ & - & - & - & $(-;-)$ \\
\hline $5-9$ & 0.2680 & 0.085 & -8.119917 & $(-17.57465 ; 1.334813)$ \\
\hline $10-14$ & 0.0783 & 0.378 & -3.9424 & $(-13.47426 ; 5.589457)$ \\
\hline $15-19$ & 0.0768 & 0.383 & 8.756811 & $(-12.62989 ; 30.14351)$ \\
\hline $20-24$ & 0.0342 & 0.565 & 5.398646 & $(-14.8236 ; 25.6209)$ \\
\hline $25-29$ & 0.0272 & 0.608 & 5.08918 & $(-16.34899 ; 26.52735)$ \\
\hline $30-34$ & 0.0013 & 0.912 & .9988316 & $(-18.54002 ; 20.53768)$ \\
\hline $35-39$ & 0.2494 & 0.098 & 9.278475 & $(-2.064558 ; 20.62151)$ \\
\hline $40-44$ & 0.1288 & 0.252 & 7.128707 & $(-5.935531 ; 20.19294)$ \\
\hline
\end{tabular}

(Continued) 
Table 1 (Continued)

\begin{tabular}{|c|c|c|c|c|}
\hline Age group (years old) & R squad** & $P^{*}$ & $\beta$ & Cl 95 \\
\hline $45-49$ & 0.1340 & 0.242 & 6.946344 & $(-5.49442 ; 19.38711)$ \\
\hline $50-54$ & 0.6170 & 0.002 & 14.59765 & $(6.494072 ; 22.70123)$ \\
\hline $55-59$ & 0.7060 & 0.001 & 13.47724 & $(7.349769 ; 19.60472)$ \\
\hline $60-64$ & 0.6329 & 0.002 & 10.50595 & $(4.868658 ; 16.14323)$ \\
\hline $65-69$ & 0.4287 & 0.021 & 8.885459 & $(1.65776 ; 16.11316)$ \\
\hline $70-74$ & 0.6737 & 0.001 & 9.83829 & $(5.013689 ; 14.66289)$ \\
\hline $75-79$ & 0.5022 & 0.010 & 8.232186 & $(2.457533 ; 14.00684)$ \\
\hline$\geq 80$ & 0.7868 & $<0.001$ & 10.1985 & $(6.457938 ; 13.93906)$ \\
\hline Total & 0.1973 & 0.148 & 1.137583 & $(-.4791081 ; 2.754273)$ \\
\hline \multicolumn{5}{|l|}{ Ceará } \\
\hline $0-4$ anos & 0.9255 & $<0.001$ & 42.20498 & (33.77054; 50.63941) \\
\hline 5-9 anos & 0.1931 & 0.153 & 4.953222 & $(-2.180051 ; 12.08649)$ \\
\hline $10-14$ anos & 0.3611 & 0.039 & -8.326455 & $(-16.13106 ;-.5218486)$ \\
\hline 15-19 anos & 0.0125 & 0.730 & 6.826819 & $(-35.95155 ; 49.60519)$ \\
\hline $20-24$ anos & 0.0010 & 0.924 & 1.668725 & $(-36.1252 ; 39.46265)$ \\
\hline $25-29$ anos & 0.0110 & 0.745 & 4.83647 & $(-27.4152 ; 37.08814)$ \\
\hline $30-34$ anos & 0.2234 & 0.121 & 18.97648 & $(-5.953305 ; 43.90627)$ \\
\hline $35-39$ anos & 0.3043 & 0.063 & 18.08948 & $(-1.181372 ; 37.36033)$ \\
\hline $40-44$ anos & 0.3546 & 0.041 & 17.00559 & $(.8398597 ; 33.17131)$ \\
\hline $45-49$ anos & 0.2897 & 0.071 & 20.09378 & $(-2.077912 ; 42.26548)$ \\
\hline $50-54$ anos & 0.7235 & $<0.001$ & 28.55369 & $(16.11681 ; 40.99056)$ \\
\hline 55-59 anos & 0.6508 & 0.002 & 18.92031 & $(9.154007 ; 28.68661)$ \\
\hline $60-64$ anos & 0.4718 & 0.014 & 11.65432 & $(2.966171 ; 20.34248)$ \\
\hline $65-69$ anos & 0.5317 & 0.007 & 17.87934 & $(6.055608 ; 29.70308)$ \\
\hline $70-74$ anos & 0.6238 & 0.002 & 13.10129 & $(5.932703 ; 20.26987)$ \\
\hline $75-79$ anos & 0.8032 & $<0.001$ & 15.22768 & $(9.916159 ; 20.53921)$ \\
\hline 80 anos e mais & 0.8001 & $<0.001$ & 11.26754 & $(7.299529 ; 15.23555)$ \\
\hline Total & 0.3674 & 0.037 & 2.429333 & $(.183355 ; 4.67531)$ \\
\hline \multicolumn{5}{|l|}{ Distrito Federal } \\
\hline $0-4$ & 0.5528 & 0.006 & 48.28478 & $(17.68379 ; 78.88576)$ \\
\hline $5-9$ & 0.3190 & 0.056 & 17.52021 & $(-.5150385 ; 35.55545)$ \\
\hline $10-14$ & 0.1987 & 0.146 & 10.8635 & $(-4.506086 ; 26.23308)$ \\
\hline $15-19$ & 0.2848 & 0.074 & -13.28613 & $(-28.12191 ; 1.54965)$ \\
\hline $20-24$ & 0.2313 & 0.113 & -23.96799 & $(-54.75799 ; 6.822001)$ \\
\hline $25-29$ & 0.3269 & 0.052 & -20.67096 & $(-41.57064 ; 0.2287196)$ \\
\hline $30-34$ & 0.1895 & 0.157 & -16.22486 & $(-39.86602 ; 7.416303)$ \\
\hline $35-39$ & 0.0061 & 0.810 & -2.959132 & $(-29.64374 ; 23.72547)$ \\
\hline $40-44$ & 0.1359 & 0.238 & -14.25478 & $(-39.57936 ; 11.0698)$ \\
\hline $45-49$ & 0.0334 & 0.570 & -7.526415 & $(-36.04835 ; 20.99552)$ \\
\hline $50-54$ & 0.0246 & 0.627 & 6.272453 & $(-21.57553 ; 34.12044)$ \\
\hline $55-59$ & 0.0351 & 0.560 & 7.980733 & $(-21.49313 ; 37.45459)$ \\
\hline $60-64$ & 0.1761 & 0.174 & -11.91843 & $(-30.08431 ; 6.247448)$ \\
\hline $65-69$ & 0.0183 & 0.675 & -4.589693 & $(-28.27049 ; 19.0911)$ \\
\hline
\end{tabular}


Table 1 (Continued)

\begin{tabular}{|c|c|c|c|c|}
\hline Age group (years old) & R squad $^{* *}$ & $P^{*}$ & $\beta$ & $\mathrm{Cl} 95$ \\
\hline $70-74$ & 0.0030 & 0.866 & 1.376055 & $(-16.28522 ; 19.03733)$ \\
\hline $75-79$ & 0.0181 & 0.677 & 3.840688 & $(-16.07957 ; 23.76094)$ \\
\hline$\geq 80$ & 0.3716 & 0.035 & 17.91251 & $(1.498385 ; 34.32663)$ \\
\hline Total & 0.0000 & 0.993 & -0.0134746 & $(-3.328418 ; 3.301469)$ \\
\hline \multicolumn{5}{|l|}{ Espírito Santo } \\
\hline $0-4$ & 0.2495 & 0.098 & 8.605444 & $(-1.910125 ; 19.12101)$ \\
\hline $5-9$ & 0.4607 & 0.015 & -11.7434 & $(-20.69503 ;-2.79177)$ \\
\hline $10-14$ & 0.5838 & 0.004 & -13.10745 & $(-20.90578 ;-5.309118)$ \\
\hline $15-19$ os & 0.0060 & 0.811 & -2.956416 & $(-29.77303 ; 23.86019)$ \\
\hline $20-24$ & 0.2565 & 0.093 & -21.90075 & $(-48.17601 ; 4.374519)$ \\
\hline $25-29$ & 0.1998 & 0.145 & -18.36249 & $(-44.25595 ; 7.530971)$ \\
\hline $30-34$ & 0.0795 & 0.375 & -10.25081 & $(-34.82746 ; 14.32584)$ \\
\hline $35-39$ & 0.0413 & 0.527 & -7.748253 & $(-34.06466 ; 18.56815)$ \\
\hline $40-44$ & 0.0629 & 0.432 & -9.351442 & $(-34.77878 ; 16.0759)$ \\
\hline $45-49$ & 0.0091 & 0.769 & 3.960317 & $(-25.22172 ; 33.14236)$ \\
\hline $50-54$ & 0.0004 & 0.950 & .7846021 & $(-26.2464 ; 27.8156)$ \\
\hline $55-59$ & 0.0163 & 0.692 & 3.529023 & $(-15.77818 ; 22.83623)$ \\
\hline $60-64$ & 0.0517 & 0.477 & 4.951812 & $(-9.993854 ; 19.89748)$ \\
\hline $65-69$ & 0.5987 & 0.003 & 17.80595 & $(7.535183 ; 28.07672)$ \\
\hline $70-74$ & 0.2782 & 0.078 & 12.88594 & $(-1.73772 ; 27.5096)$ \\
\hline $75-79$ & 0.3922 & 0.029 & 13.28996 & $(1.633018 ; 24.94691)$ \\
\hline$\geq 80$ & 0.6340 & 0.002 & 12.72639 & $(5.912973 ; 19.53982)$ \\
\hline Total & 0.0022 & 0.884 & -0.1688155 & $(-2.675465 ; 2.337834)$ \\
\hline \multicolumn{5}{|l|}{ Goiás } \\
\hline $0-4$ & 0.3730 & 0.035 & 9.210891 & $(.7973597 ; 17.62442)$ \\
\hline $5-9$ & 0.1719 & 0.180 & -4.726419 & $(-12.03689 ; 2.584047)$ \\
\hline $10-14$ & 0.6916 & 0.001 & -10.02397 & $(-14.74016 ;-5.307778)$ \\
\hline $15-19$ & 0.5722 & 0.004 & -18.00162 & $(-28.96969 ;-7.033548)$ \\
\hline $20-24$ & 0.6801 & 0.001 & -19.42995 & $(-28.81834 ;-10.04156)$ \\
\hline $25-29$ & 0.4230 & 0.022 & -17.15218 & $(-31.26806 ;-3.036304)$ \\
\hline $30-34$ & 0.2811 & 0.076 & -9.407014 & $(-20.00648 ; 1.192457)$ \\
\hline $35-39$ & 0.1819 & 0.167 & -6.438802 & $(-16.05991 ; 3.182308)$ \\
\hline $40-44$ & 0.0020 & 0.889 & -.8957415 & $(-14.8523 ; 13.06082)$ \\
\hline $45-49$ & 0.0210 & 0.653 & 2.40813 & $(-9.180696 ; 13.99696)$ \\
\hline $50-54$ & 0.0630 & 0.431 & 4.267537 & $(-7.331111 ; 15.86619)$ \\
\hline $55-59$ & 0.0061 & 0.810 & 1.197741 & $(-9.591894 ; 11.98738)$ \\
\hline $60-64$ & 0.7146 & 0.001 & 18.88126 & $(10.47422 ; 27.2883)$ \\
\hline $65-69$ & 0.2395 & 0.106 & 6.455586 & $(-1.649954 ; 14.56113)$ \\
\hline $70-74$ & 0.6730 & 0.001 & 18.32539 & $(9.32532 ; 27.32547)$ \\
\hline $75-79$ & 0.6243 & 0.002 & 19.59943 & $(8.887206 ; 30.31166)$ \\
\hline$\geq 80$ & 0.7724 & $<0.001$ & 17.07358 & $(10.54316 ; 23.604)$ \\
\hline Total & 0.0051 & 0.825 & .1134388 & $(-1.003239 ; 1.230116)$ \\
\hline
\end{tabular}


310 Brazilian Incidence of TBI Takahashi et al.

Table 1 (Continued)

\begin{tabular}{|c|c|c|c|c|}
\hline Age group (years old) & R squad ${ }^{* *}$ & $P^{*}$ & $\beta$ & Cl 95 \\
\hline \multicolumn{5}{|l|}{ Maranhão } \\
\hline $0-4$ & 0.4083 & 0.025 & 13.40847 & $(2.036438 ; 24.7805)$ \\
\hline $5-9$ & 0.3564 & 0.040 & 9.084977 & $(0.4821196 ; 17.68783)$ \\
\hline $10-14$ & 0.3616 & 0.039 & 8.37904 & $(0.5342843 ; 16.2238)$ \\
\hline $15-19$ & 0.7583 & $<0.001$ & 55.00458 & $(33.12122 ; 76.88794)$ \\
\hline $20-24$ & 0.8263 & $<0.001$ & 56.40574 & $(38.1846 ; 74.62689)$ \\
\hline $25-29$ & 0.7626 & $<0.001$ & 54.83187 & $(33.27512 ; 76.38862)$ \\
\hline $30-34$ & 0.8773 & $<0.001$ & 45.05807 & $(33.18498 ; 56.93116)$ \\
\hline $35-39$ & 0.8851 & - & - & $(-;-)$ \\
\hline $40-44$ & - & $<0.001$ & 44.77721 & (33.40696; 56.14745) \\
\hline $45-49$ & 0.9015 & $<0.001$ & 39.74757 & $(30.48813 ; 49.00702)$ \\
\hline $50-54$ & 0.9000 & $<0.001$ & 34.90037 & $(26.70416 ; 43.09658)$ \\
\hline 55-59 & 0.8044 & $<0.001$ & 27.21981 & $(17.76357 ; 36.67605)$ \\
\hline $60-64$ & 0.6769 & $<0.001$ & 21.77253 & $(11.1726 ; 32.37246)$ \\
\hline 65-69 & 0.8087 & $<0.001$ & 25.08413 & $(16.48673 ; 33.68153)$ \\
\hline $70-74$ & 0.8528 & $<0.001$ & 26.66924 & $(18.86176 ; 34.47672)$ \\
\hline $75-79$ & 0.8586 & $<0.001$ & 19.27253 & $(13.76119 ; 24.78388)$ \\
\hline$\geq 80$ & 0.8549 & $<0.001$ & 15.48653 & $(10.99124 ; 19.98183)$ \\
\hline Total & 0.9389 & $<0.001$ & 5.384739 & $(4.416786 ; 6.352693)$ \\
\hline \multicolumn{5}{|l|}{ Mato Grosso } \\
\hline 0a 4 & 0.5591 & 0.005 & 18.7699 & $(7.025052 ; 30.51476)$ \\
\hline $5-9$ & 0.4416 & 0.018 & -12.65769 & $(-22.68686 ;-2.628515)$ \\
\hline $10-14$ & 0.2833 & 0.075 & -10.38622 & $(-22.02477 ; 1.252334)$ \\
\hline $15-19$ & 0.0091 & 0.768 & 3.186886 & $(-20.20129 ; 26.57506)$ \\
\hline $20-24$ & 0.4619 & 0.015 & -34.71405 & $(-61.11214 ;-8.315949)$ \\
\hline $25-29$ & 0.0077 & 0.786 & -2.607459 & $(-23.46181 ; 18.24689)$ \\
\hline $30-34$ & - & - & - & $(-;-)$ \\
\hline $35-39$ & 0.0247 & 0.626 & -5.032936 & $(-27.30889 ; 17.24301)$ \\
\hline $40-44$ & 0.0388 & 0.539 & -4.795879 & $(-21.61225 ; 12.0205)$ \\
\hline $45-49$ & 0.0047 & 0.832 & 1.520364 & $(-14.03587 ; 17.0766)$ \\
\hline $50-54$ & 0.0070 & 0.796 & -1.401018 & $(-13.14505 ; 10.34302)$ \\
\hline $55-59$ & 0.1308 & 0.248 & 6.210128 & $(-5.070401 ; 17.49066)$ \\
\hline $60-64$ & 0.0584 & 0.449 & 7.322759 & $(-13.40256 ; 28.04808)$ \\
\hline $65-69$ & 0.0800 & 0.373 & 6.411983 & $(-8.909456 ; 21.73342)$ \\
\hline $70-74$ & 0.5258 & 0.008 & 20.48129 & $(6.775247 ; 34.18733)$ \\
\hline $75-79$ & 0.1568 & 0.203 & 9.408353 & $(-5.965013 ; 24.78172)$ \\
\hline$\geq 80$ & 0.2589 & 0.091 & 6.591994 & $(-1.265852 ; 14.44984)$ \\
\hline Total & 0.0000 & 0.999 & 0.0013807 & $(-1.912672 ; 1.915433)$ \\
\hline \multicolumn{5}{|l|}{ Mato Grosso do Sul } \\
\hline $0-4$ & 0.0197 & 0.664 & 3.42112 & $(-13.583 ; 20.42524)$ \\
\hline $5-9$ & 0.4857 & 0.012 & -16.37614 & $(-28.24894 ;-4.503346)$ \\
\hline $10-14$ & 0.0312 & 0.583 & -2.337702 & $(-11.5185 ; 6.843096)$ \\
\hline $15-19$ & 0.0470 & 0.499 & -6.845818 & $(-28.56967 ; 14.87803)$ \\
\hline
\end{tabular}


Table 1 (Continued)

\begin{tabular}{|c|c|c|c|c|}
\hline Age group (years old) & R squad** & $P^{*}$ & $\beta$ & $\mathrm{Cl} 95$ \\
\hline $20-24$ & 0.0777 & 0.380 & -8.730684 & $(-29.92216 ; 12.46079)$ \\
\hline $25-29$ & 0.2756 & 0.080 & -23.87068 & $(-51.14018 ; 3.398824)$ \\
\hline $30-34$ & 0.1372 & 0.236 & -12.84976 & $(-35.55432 ; 9.854804)$ \\
\hline $35-39$ & 0.0001 & 0.978 & -.2373176 & $(-18.58611 ; 18.11148)$ \\
\hline $40-44$ & 0.1559 & 0.204 & -10.23964 & $(-27.028 ; 6.548727)$ \\
\hline $45-49$ & 0.0252 & 0.622 & 2.97225 & $(-10.04937 ; 15.99387)$ \\
\hline $50-54$ & 0.0245 & 0.627 & -3.059208 & $(-16.65438 ; 10.53596)$ \\
\hline $55-59$ & 0.1390 & 0.233 & 9.67001 & $(-7.291056 ; 26.63108)$ \\
\hline $60-64$ & 0.1215 & 0.267 & 9.08854 & $(-8.130843 ; 26.30792)$ \\
\hline $65-69$ & 0.2607 & 0.090 & 16.14349 & $(-3.01254 ; 35.29952)$ \\
\hline $70-74$ & 0.3556 & 0.041 & 17.94235 & $(0.9225824 ; 34.96212)$ \\
\hline $75-79$ & 0.3285 & 0.051 & 12.97876 & $(-0.0945184 ; 26.05204)$ \\
\hline$\geq 80$ & 0.8016 & $<0.001$ & 19.00386 & $(12.34153 ; 25.66619)$ \\
\hline Total & 0.0006 & 0.941 & .0667343 & $(-1.893257 ; 2.026726)$ \\
\hline \multicolumn{5}{|l|}{ Minas Gerais } \\
\hline $0-4$ & 0.1821 & 0.167 & 6.961586 & $(-3.435487 ; 17.35866)$ \\
\hline $5-9$ & 0.3518 & 0.042 & -6.873006 & $(-13.44667 ;-.2993408)$ \\
\hline $10-14$ & 0.7957 & $<0.001$ & -13.08791 & $(-17.76114 ;-8.414676)$ \\
\hline $15-19$ & 0.1053 & 0.303 & -5.992335 & $(-18.30038 ; 6.315707)$ \\
\hline $20-24$ & 0.4254 & 0.022 & -12.05901 & $(-21.93353 ;-2.18449)$ \\
\hline $25-29$ & 0.3025 & 0.064 & -9.181918 & $(-19.00538 ; .6415395)$ \\
\hline $30-34$ & 0.6510 & 0.002 & -13.43748 & $(-20.37043 ;-6.50453)$ \\
\hline $35-39$ & 0.0736 & 0.394 & -2.554975 & $(-8.939744 ; 3.829794)$ \\
\hline $40-44$ & 0.0383 & 0.542 & 1.991558 & $(-5.037854 ; 9.02097)$ \\
\hline $45-49$ & 0.0701 & 0.406 & 2.364434 & $(-3.705249 ; 8.434118)$ \\
\hline $50-54$ & 0.5473 & 0.006 & 7.337322 & $(2.635892 ; 12.03875)$ \\
\hline $55-59$ & 0.7332 & $<0.001$ & 12.63894 & $(7.267076 ; 18.01081)$ \\
\hline $60-64$ & 0.6021 & 0.003 & 9.05285 & $(3.867699 ; 14.238)$ \\
\hline $65-69$ & 0.7943 & $<0.001$ & 11.15101 & $(7.152702 ; 15.14931)$ \\
\hline $70-74$ & - & - & - & $(-;-)$ \\
\hline $75-79$ & 0.7754 & $<0.001$ & 11.06995 & $(6.872554 ; 15.26734)$ \\
\hline$\geq 80$ & 0.9356 & $<0.001$ & 14.06956 & $(11.46953 ; 16.6696)$ \\
\hline Total & 0.0835 & 0.362 & 0.2751456 & $(-0.3672378 ; 0.9175289$ \\
\hline \multicolumn{5}{|l|}{ Pará } \\
\hline $0-4$ & 0.7933 & $<0.001$ & 21.84819 & $(13.99122 ; 29.70516)$ \\
\hline $5-9$ & 0.3665 & 0.037 & 5.924406 & $(.4363277 ; 11.41248)$ \\
\hline $10-14$ & 0.2175 & 0.126 & 6.177389 & $(-2.077839 ; 14.43262)$ \\
\hline $15-19$ & 0.4179 & 0.023 & 32.01537 & $(5.390975 ; 58.63977)$ \\
\hline $20-24$ & 0.4921 & 0.011 & 35.32087 & $(10.03745 ; 60.6043)$ \\
\hline $25-29$ & 0.2174 & 0.127 & 23.45804 & $(-7.903151 ; 54.81922)$ \\
\hline $30-34$ & 0.2684 & 0.084 & 22.75911 & $(-3.718781 ; 49.237)$ \\
\hline $35-39$ & 0.5816 & 0.004 & 29.55384 & $(11.89267 ; 47.21502)$ \\
\hline $40-44$ & 0.6504 & 0.002 & 23.17685 & $(11.20419 ; 35.1495)$ \\
\hline
\end{tabular}

(Continued) 
312 Brazilian Incidence of TBI Takahashi et al.

Table 1 (Continued)

\begin{tabular}{|c|c|c|c|c|}
\hline Age group (years old) & R squad** & $P^{*}$ & $\beta$ & Cl 95 \\
\hline $45-49$ & 0.7736 & $<0.001$ & 26.46024 & $(16.37502 ; 36.54547)$ \\
\hline $50-54$ & 0.5886 & 0.004 & 17.85489 & $(7.338146 ; 28.37163)$ \\
\hline $55-59$ & 0.6247 & - & - & $(-;-)$ \\
\hline $60-64$ & - & 0.002 & 15.76658 & $(7.155296 ; 24.37786)$ \\
\hline $65-69$ & 0.5293 & 0.007 & 10.33715 & $(3.468329 ; 17.20598)$ \\
\hline $70-74$ & 0.2305 & 0.114 & 6.495517 & $(-1.867382 ; 14.85842)$ \\
\hline $75-79$ & 0.5831 & 0.004 & 11.3059 & (4.569893; 18.04191) \\
\hline$\geq 80$ & 0.6372 & 0.002 & 9.641141 & $(4.515114 ; 14.76717)$ \\
\hline Total & 0.5704 & 0.005 & 3.059595 & $(1.188541 ; 4.930649)$ \\
\hline \multicolumn{5}{|l|}{ Paraíba } \\
\hline $0-4$ & 0.4742 & 0.013 & -35.99264 & $(-62.69471 ;-9.290564)$ \\
\hline $5-9$ & 0.6930 & 0.001 & -38.71377 & $(-56.87086 ;-20.55669)$ \\
\hline $10-14$ & 0.6678 & 0.001 & -39.00649 & $(-58.39056 ;-19.62242)$ \\
\hline $15-19$ & 0.7003 & 0.001 & -87.61793 & $(-128.0089 ;-47.22699)$ \\
\hline $20-24$ & 0.6987 & 0.001 & -92.7525 & $(-135.6656 ;-49.83945)$ \\
\hline $25-29$ & 0.6665 & 0.001 & -83.84856 & $(-125.6361 ;-42.061)$ \\
\hline $30-34$ & 0.7276 & $<0.001$ & -70.63522 & $(-101.091 ;-40.17941)$ \\
\hline $35-39$ & 0.7657 & $<0.001$ & -67.82586 & $(-94.25835 ;-41.39336)$ \\
\hline $40-44$ & 0.6273 & 0.002 & -44.03293 & $(-67.94999 ;-20.11588)$ \\
\hline $45-49$ & 0.5887 & 0.004 & -36.91202 & $(-58.65259 ;-15.17145)$ \\
\hline $50-54$ & 0.4293 & 0.021 & -28.51988 & $(-51.68832 ;-5.351439)$ \\
\hline $55-59$ & 0.5995 & 0.003 & -21.73153 & $(-34.24592 ;-9.217132)$ \\
\hline $60-64$ & 0.5604 & 0.005 & -24.0388 & $(-39.03881 ;-9.038789)$ \\
\hline $65-69$ & 0.4485 & 0.017 & -15.63875 & $(-27.85832 ;-3.419171)$ \\
\hline $70-74$ & 0.5616 & 0.005 & -14.20204 & $(-23.04254 ;-5.36154)$ \\
\hline $75-79$ & 0.4731 & 0.013 & -12.52148 & $(-21.83144 ;-3.211509)$ \\
\hline$\geq 80$ & 0.2718 & 0.082 & -8.599393 & $(-18.51825 ; 1.319466)$ \\
\hline Total & 0.6963 & 0.001 & -7.225898 & $(-10.58822 ;-3.863579)$ \\
\hline \multicolumn{5}{|l|}{ Paraná } \\
\hline $0-4$ & 0.4938 & 0.011 & 37.26343 & $(10.68098 ; 63.84587)$ \\
\hline $5-9$ & 0.0545 & 0.465 & -5.110262 & $(-20.11331 ; 9.89279)$ \\
\hline $10-14$ & 0.4548 & 0.016 & -16.30144 & $(-28.87669 ;-3.726195)$ \\
\hline $15-19$ & 0.0980 & 0.322 & -12.57734 & $(-39.4572 ; 14.30251)$ \\
\hline $20-24$ & 0.0225 & 0.642 & -4.754975 & $(-26.83866 ; 17.32871)$ \\
\hline $25-29$ & 0.0439 & 0.514 & -4.132378 & $(-17.72856 ; 9.4638)$ \\
\hline $30-34$ & 0.1196 & 0.271 & -9.18386 & $(-26.74326 ; 8.375539)$ \\
\hline $35-39$ & 0.2290 & 0.116 & 9.932479 & $(-2.907918 ; 22.77288)$ \\
\hline $40-44$ & 0.1718 & 0.180 & 7.846936 & $(-4.291423 ; 19.9853)$ \\
\hline $45-49$ & 0.4261 & 0.021 & 13.53267 & $(2.467152 ; 24.59819)$ \\
\hline $50-54$ & 0.8138 & $<0.001$ & 22.97141 & $(15.22881 ; 30.71402)$ \\
\hline $55-59$ & 0.7691 & $<0.001$ & 26.91298 & $(16.52331 ; 37.30264)$ \\
\hline $60-64$ & 0.8907 & $<0.001$ & 18.99117 & $(14.30481 ; 23.67752)$ \\
\hline $65-69$ & 0.8290 & $<0.001$ & 20.51924 & (13.95269; 27.08579) \\
\hline
\end{tabular}


Table 1 (Continued)

\begin{tabular}{|c|c|c|c|c|}
\hline Age group (years old) & R squad** & $P^{*}$ & $\beta$ & Cl 95 \\
\hline $70-74$ & 0.9027 & $<0.001$ & 26.11186 & $(20.07215 ; 32.15157)$ \\
\hline $75-79$ & 0.7660 & $<0.001$ & 19.94069 & $(12.17498 ; 27.70639)$ \\
\hline$\geq 80$ & 0.9277 & $<0.001$ & 24.35314 & $(19.56269 ; 29.14359)$ \\
\hline Total & 0.4158 & 0.024 & 1.763157 & $(0.2904611 ; 3.235854)$ \\
\hline \multicolumn{5}{|l|}{ Pernambuco } \\
\hline $0-4$ & 0.2961 & 0.067 & 18.14129 & $(-1.566685 ; 37.84927)$ \\
\hline $5-9$ & 0.3267 & 0.052 & -8.088987 & $(-16.27101 ; 0.0930329)$ \\
\hline $10-14$ & 0.4377 & 0.019 & -10.57062 & $(-19.01214 ;-2.129102)$ \\
\hline 15-19 & 0.0860 & 0.355 & 12.28324 & $(-15.92832 ; 40.49481)$ \\
\hline $20-24$ & 0.0903 & 0.343 & 16.19489 & $(-20.02578 ; 52.41556)$ \\
\hline $25-29$ & 0.0973 & 0.324 & 10.80126 & $(-12.37683 ; 33.97935)$ \\
\hline $30-34$ & 0.0256 & 0.619 & 5.42487 & $(-18.15235 ; 29.00209)$ \\
\hline $35-39$ & 0.1225 & 0.265 & 8.5803 & $(-7.59937 ; 24.75997)$ \\
\hline $40-44$ & 0.1783 & 0.172 & 9.634181 & $(-4.940764 ; 24.20913)$ \\
\hline $45-49$ & 0.1557 & 0.204 & 9.533999 & $(-6.11064 ; 25.17864)$ \\
\hline $50-54$ & 0.1002 & 0.316 & 6.663475 & $(-7.402355 ; 20.7293)$ \\
\hline $55-59$ & 0.4510 & 0.017 & 10.13703 & $(2.256587 ; 18.01748)$ \\
\hline $60-64$ & 0.4023 & 0.027 & 14.35866 & $(2.026321 ; 26.691)$ \\
\hline $65-69$ & 0.3257 & 0.053 & 11.03945 & $(-0.1514642 ; 22.23037)$ \\
\hline $70-74$ & 0.7400 & $<0.001$ & 15.21134 & $(8.858736 ; 21.56394)$ \\
\hline $75-79$ & 0.4535 & 0.016 & 9.951957 & $(2.254806 ; 17.64911)$ \\
\hline$\geq 80$ & 0.7151 & 0.001 & 10.0646 & $(5.587967 ; 14.54124)$ \\
\hline Total & 0.1768 & 0.174 & 1.49361 & $(-0.7773257 ; 3.764545)$ \\
\hline \multicolumn{5}{|l|}{ Piauí } \\
\hline $0-4$ & 0.2296 & 0.115 & 6.075597 & $(-1.765201 ; 13.91639)$ \\
\hline $5-9$ & 0.0004 & 0.951 & -0.2927463 & $(-10.68402 ; 10.09853)$ \\
\hline $10-14$ & 0.0471 & 0.498 & 3.380113 & $(-7.337003 ; 14.09723)$ \\
\hline $15-19$ & 0.2785 & 0.078 & 40.48692 & $(-5.433354 ; 86.4072)$ \\
\hline $20-24$ & 0.6509 & 0.002 & 63.43446 & (30.70179; 96.16714) \\
\hline $25-29$ & 0.4999 & 0.010 & 55.80504 & $(16.47574 ; 95.13434)$ \\
\hline $30-34$ & 0.6292 & 0.002 & 52.08474 & $(23.91067 ; 80.2588)$ \\
\hline $35-39$ & 0.6306 & 0.002 & 48.24226 & $(22.22772 ; 74.25681)$ \\
\hline $40-44$ & 0.7516 & $<0.001$ & 57.07575 & $(33.95903 ; 80.19247)$ \\
\hline $45-49$ & 0.7483 & $<0.001$ & 40.42415 & $(23.90564 ; 56.94265)$ \\
\hline $50-54$ & 0.7788 & $<0.001$ & 40.06484 & $(25.01891 ; 55.11077)$ \\
\hline $55-59$ & 0.6599 & 0.001 & 25.97404 & $(12.8361 ; 39.11198)$ \\
\hline $60-64$ & 0.5391 & 0.007 & 20.49497 & $(7.143766 ; 33.84618)$ \\
\hline 65-69 & 0.6338 & 0.002 & 28.70731 & $(13.33298 ; 44.08164)$ \\
\hline $70-74$ & 0.6275 & 0.002 & 25.38844 & $(11.60576 ; 39.17111)$ \\
\hline $75-79$ & - & - & - & $(-;-)$ \\
\hline$\geq 80$ & 0.6185 & 0.002 & 15.00641 & $(6.701436 ; 23.31138)$ \\
\hline Total & 0.7308 & $<0.001$ & 5.380512 & $(3.079627 ; 7.681396)$ \\
\hline
\end{tabular}


314 Brazilian Incidence of TBI Takahashi et al.

Table 1 (Continued)

\begin{tabular}{|c|c|c|c|c|}
\hline Age group (years old) & R squad** & $P^{*}$ & $\beta$ & Cl 95 \\
\hline \multicolumn{5}{|l|}{ Rio de Janeiro } \\
\hline $0-4$ & 0.7448 & $<0.001$ & 31.26287 & $(18.3676 ; 44.15815)$ \\
\hline $5-9$ & 0.0181 & 0.677 & 1.252736 & $(-5.253708 ; 7.759181)$ \\
\hline $10-14$ & 0.0251 & 0.623 & 1.805437 & $(-6.130486 ; 9.741359)$ \\
\hline $15-19$ & 0.3805 & 0.033 & 13.74597 & $(1.387978 ; 26.10397)$ \\
\hline $20-24$ & 0.5775 & 0.004 & 22.64667 & (8.997514; 36.29582) \\
\hline $25-29$ & 0.5605 & 0.005 & 14.21013 & $(5.344407 ; 23.07585)$ \\
\hline $30-34$ & 0.4974 & 0.010 & 11.23959 & $(3.278907 ; 19.20027)$ \\
\hline $35-39$ & 0.7979 & $<0.001$ & 17.20162 & $(11.10217 ; 23.30107)$ \\
\hline $40-44$ & 0.5798 & 0.004 & 10.84132 & $(4.338807 ; 17.34382)$ \\
\hline $45-49$ & 0.6990 & 0.001 & 14.02548 & $(7.540148 ; 20.51081)$ \\
\hline $50-54$ & 0.7519 & $<0.001$ & 15.97452 & $(9.508284 ; 22.44076)$ \\
\hline $55-59$ & 0.8127 & $<0.001$ & 17.71869 & $(11.72554 ; 23.71184)$ \\
\hline $60-64$ & 0.9534 & $<0.001$ & 17.55152 & $(14.81644 ; 20.28659)$ \\
\hline $65-69$ & 0.9487 & $<0.001$ & 20.26889 & $(16.94838 ; 23.58941)$ \\
\hline $70-74$ & 0.9336 & $<0.001$ & 16.62608 & $(13.5008 ; 19.75135)$ \\
\hline $75-79$ & 0.9227 & $<0.001$ & 14.33734 & $(11.41289 ; 17.26178)$ \\
\hline$\geq 80$ & 0.8565 & $<0.001$ & 12.006 & $(8.543407 ; 15.46859)$ \\
\hline Total & 0.7933 & $<0.001$ & 2.527149 & $(1.618105 ; 3.436192)$ \\
\hline \multicolumn{5}{|l|}{ Rio Grande do Norte } \\
\hline $0-4$ & 0.6042 & 0.003 & 11.86768 & $(5.099782 ; 18.63558)$ \\
\hline $5-9$ & 0.0027 & 0.873 & .2571334 & $(-3.241539 ; 3.755806)$ \\
\hline $10-14$ & 0.0214 & 0.650 & 1.438548 & $(-5.421406 ; 8.298502)$ \\
\hline $15-19$ & 0.1533 & 0.208 & 9.421095 & $(-6.182316 ; 25.02451)$ \\
\hline $20-24$ & 0.0140 & 0.714 & 4.433893 & $(-21.79364 ; 30.66142)$ \\
\hline $25-29$ & 0.0055 & 0.818 & -2.167655 & $(-22.65635 ; 18.32104)$ \\
\hline $30-34$ & 0.1047 & 0.305 & 8.272677 & $(-8.770621 ; 25.31597)$ \\
\hline $35-39$ & 0.1014 & 0.313 & 8.271281 & $(-9.079859 ; 25.62242)$ \\
\hline $40-44$ & - & - & - & $(-;-)$ \\
\hline $45-49$ & 0.3546 & 0.041 & 16.5471 & $(.8189716 ; 32.27522)$ \\
\hline $50-54$ & 0.6948 & 0.001 & 20.65614 & $(11.00932 ; 30.30296)$ \\
\hline $55-59$ & 0.4065 & 0.026 & 16.56427 & $(2.462803 ; 30.66573)$ \\
\hline $60-64$ & 0.1065 & 0.301 & 5.646568 & $(-5.878394 ; 17.17153)$ \\
\hline $65-69$ & 0.5202 & 0.008 & 15.37487 & $(4.970457 ; 25.77928)$ \\
\hline $70-74$ & 0.5093 & 0.009 & 17.87559 & $(5.512058 ; 30.23912)$ \\
\hline $75-79$ & 0.4007 & 0.027 & 7.670452 & $(1.061139 ; 14.27976)$ \\
\hline$\geq 80$ & 0.5948 & 0.003 & 10.88143 & $(4.553646 ; 17.20922)$ \\
\hline Total & 0.3578 & 0.040 & 1.630702 & $(0.0913046 ; 3.170099)$ \\
\hline \multicolumn{5}{|l|}{ Rio Grande do Sul } \\
\hline $0-4$ & 0.6969 & 0.001 & -22.05091 & $(-32.29797 ;-11.80386)$ \\
\hline $5-9$ & 0.8422 & $<0.001$ & -29.96878 & $(-39.10971 ;-20.82785)$ \\
\hline $10-14$ & 0.9256 & $<0.001$ & -29.20466 & $(-35.03851 ;-23.37081)$ \\
\hline $15-19$ & 0.7185 & $<0.001$ & -26.69353 & $(-38.46568 ;-14.92137)$ \\
\hline $20-24$ & 0.7218 & $<0.001$ & -34.53079 & $(-49.63449 ;-19.42709)$ \\
\hline
\end{tabular}


Table 1 (Continued)

\begin{tabular}{|c|c|c|c|c|}
\hline Age group (years old) & $\mathrm{R}_{\text {squad }}$ s* $^{2}$ & $P^{*}$ & $\beta$ & $\mathrm{Cl} 95$ \\
\hline $25-29$ & 0.7947 & $<0.001$ & -20.61702 & $(-28.00003 ;-13.234)$ \\
\hline $30-34$ & 0.8291 & $<0.001$ & -19.39893 & $(-25.60559 ;-13.19226)$ \\
\hline $35-39$ & 0.6833 & 0.001 & -14.8858 & $(-22.02558 ;-7.746012)$ \\
\hline $40-44$ & 0.7851 & $<0.001$ & -19.83451 & $(-27.14641 ;-12.52262)$ \\
\hline $45-49$ & 0.6811 & 0.001 & -17.85139 & $(-26.45821 ;-9.244562)$ \\
\hline $50-54$ & 0.3599 & 0.039 & -7.119917 & $(-13.8099 ;-.4299314)$ \\
\hline $55-59$ & 0.0109 & 0.746 & -0.743476 & $(-5.724769 ; 4.237817)$ \\
\hline $60-64$ & 0.0000 & 0.988 & -0.0284309 & $(-4.074809 ; 4.017947)$ \\
\hline $65-69$ & 0.0255 & 0.620 & -0.8417308 & $(-4.50924 ; 2.825778)$ \\
\hline $70-74$ & 0.5248 & 0.008 & 6.759869 & $(2.22781 ; 11.29193)$ \\
\hline $75-79$ & 0.3979 & 0.028 & 5.220151 & $(0.6959262 ; 9.744376)$ \\
\hline$\geq \mathrm{m} 80$ & 0.7898 & $<0.001$ & 4.562996 & $(2.904476 ; 6.221517)$ \\
\hline Total & 0.8132 & $<0.001$ & -2.272268 & $(-3.039517 ;-1.505019)$ \\
\hline \multicolumn{5}{|l|}{ Rondônia } \\
\hline $0-4$ & 0.3054 & 0.062 & 43.02182 & $(-2.69612 ; 88.73976)$ \\
\hline $5-9$ & 0.0094 & 0.765 & 3.247585 & $(-20.26745 ; 26.76262)$ \\
\hline $10-14$ & 0.0014 & 0.907 & -0.6795796 & $(-13.31137 ; 11.95221)$ \\
\hline $15-19$ & 0.0423 & 0.521 & -8.948426 & $(-38.93841 ; 21.04156)$ \\
\hline $20-24$ & 0.0092 & 0.766 & 5.734106 & $(-36.08463 ; 47.55284)$ \\
\hline $25-29$ & 0.2131 & 0.131 & -25.45637 & $(-59.91858 ; 9.005836)$ \\
\hline $30-34$ & 0.0053 & 0.822 & -3.547277 & $(-37.84045 ; 30.7459)$ \\
\hline $35-39$ & 0.0023 & 0.883 & -2.2987 & $(-36.11083 ; 31.51343)$ \\
\hline $40-44$ & 0.0000 & 0.988 & .2421229 & $(-34.24932 ; 34.73356)$ \\
\hline $45-49$ & 0.0502 & 0.484 & 10.55388 & $(-21.77585 ; 42.88362)$ \\
\hline $50-54$ & 0.0516 & 0.478 & 10.42449 & $(-21.08054 ; 41.92951)$ \\
\hline 55-59 & 0.1352 & 0.240 & 16.99913 & $(-13.29733 ; 47.29559)$ \\
\hline $60-64$ & 0.0314 & 0.582 & 7.512004 & $(-21.89156 ; 36.91557)$ \\
\hline $65-69$ & 0.5055 & 0.010 & 26.1204 & $(7.915564 ; 44.32524)$ \\
\hline $70-74$ & 0.3207 & 0.055 & 17.58742 & $(-0.4462037 ; 35.62104)$ \\
\hline $75-79$ & 0.7654 & $<0.001$ & 21.1701 & $(12.91084 ; 29.42936)$ \\
\hline$\geq 80$ & 0.6408 & 0.002 & 24.77988 & $(11.70642 ; 37.85334)$ \\
\hline Total & 0.0716 & 0.401 & 1.464626 & $(-2.252476 ; 5.181728)$ \\
\hline \multicolumn{5}{|l|}{ Roraima } \\
\hline $0-4$ & 0.0277 & 0.605 & 12.86429 & $(-40.86586 ; 66.59444)$ \\
\hline $5-9$ & 0.1175 & 0.275 & -21.05591 & $(-61.71881 ; 19.607)$ \\
\hline $10-14$ & 0.0096 & 0.762 & 3.5116 & $(-21.58209 ; 28.60529)$ \\
\hline $15-19$ & 0.0006 & 0.938 & 1.060959 & $(-28.37494 ; 30.49686)$ \\
\hline $20-24$ & 0.0556 & 0.461 & 8.188362 & $(-15.58648 ; 31.96321)$ \\
\hline $25-29$ & 0.0289 & 0.598 & 12.45662 & $(-38.44482 ; 63.35805)$ \\
\hline $30-34$ & 0.0430 & 0.518 & 11.47874 & $(-26.66178 ; 49.61926)$ \\
\hline $35-39$ & 0.0000 & 0.988 & -.3329741 & $(-46.70785 ; 46.0419)$ \\
\hline $40-44$ & 0.0747 & 0.390 & 14.91072 & $(-22.06766 ; 51.8891)$ \\
\hline $45-49$ & 0.0823 & 0.366 & -16.0201 & $(-53.72331 ; 21.68311)$ \\
\hline
\end{tabular}

(Continued) 
Table 1 (Continued)

\begin{tabular}{|c|c|c|c|c|}
\hline Age group (years old) & R squad $^{* *}$ & $P^{*}$ & $\beta$ & $\mathrm{Cl} 95$ \\
\hline $50-54$ & 0.0004 & 0.952 & -.9046439 & $(-33.77657 ; 31.96728)$ \\
\hline $55-59$ & 0.2772 & 0.079 & 19.0456 & $(-2.62515 ; 40.71635)$ \\
\hline $60-64$ & 0.1216 & 0.267 & 19.01485 & $(-16.99041 ; 55.02012)$ \\
\hline $65-69$ & 0.1013 & 0.313 & 21.23019 & $(-23.31962 ; 65.78)$ \\
\hline $70-74$ & 0.3070 & 0.062 & 21.60614 & $(-1.267012 ; 44.4793)$ \\
\hline $75-79$ & 0.2282 & 0.116 & 53.43766 & $(-15.81028 ; 122.6856)$ \\
\hline$\geq 80$ & 0.4808 & 0.012 & 31.04696 & $(8.315454 ; 53.77847)$ \\
\hline Total & 0.2334 & 0.112 & 1.915391 & $(-.5304348 ; 4.361216)$ \\
\hline \multicolumn{5}{|l|}{ Santa Catarina } \\
\hline $0-4$ & 0.1563 & 0.203 & 10.17735 & $(-6.481488 ; 26.83619)$ \\
\hline $5-9$ & 0.2460 & 0.101 & -8.157149 & $(-18.21996 ; 1.905664)$ \\
\hline $10-14$ & 0.5858 & 0.004 & -14.32318 & $(-22.80979 ;-5.836566)$ \\
\hline $15-19$ & 0.4687 & 0.014 & -24.34532 & $(-42.6102 ;-6.080434)$ \\
\hline $20-24$ & 0.4766 & 0.013 & -24.87304 & $(-43.238 ;-6.508085)$ \\
\hline $25-29$ & 0.2841 & 0.074 & -11.10234 & $(-23.52064 ; 1.315954)$ \\
\hline $30-34$ & 0.3182 & 0.056 & -11.73384 & $(-23.83483 ; .367142)$ \\
\hline $35-39$ & 0.2070 & 0.137 & -6.471365 & $(-15.39588 ; 2.453153)$ \\
\hline $40-44$ & - & - & - & $(-;-)$ \\
\hline $45-49$ & 0.3714 & 0.035 & -8.851694 & $(-16.96595 ;-0.7374419)$ \\
\hline $50-54$ & 0.0139 & 0.716 & 2.095916 & $(-10.36343 ; 14.55526)$ \\
\hline $55-59$ & 0.1078 & 0.297 & 3.897368 & $(-4.000752 ; 11.79549)$ \\
\hline $60-64$ & 0.3265 & 0.052 & 10.36606 & $(-0.1252432 ; 20.85736)$ \\
\hline $65-69$ & 0.2089 & 0.135 & 6.724965 & $(-2.496781 ; 15.94671)$ \\
\hline $70-74$ & 0.3337 & 0.049 & 3.852307 & $(.0168896 ; 7.687725)$ \\
\hline $75-79$ & 0.6821 & 0.001 & 13.79607 & $(7.159966 ; 20.43218)$ \\
\hline$\geq 80$ & 0.5499 & 0.006 & 10.76667 & $(3.903044 ; 17.6303)$ \\
\hline Total & 0.0972 & 0.324 & -.5525308 & $(-1.739186 ; .6341243)$ \\
\hline \multicolumn{5}{|l|}{ São Paulo } \\
\hline $0-4$ & 0.4019 & 0.027 & -18.84617 & $(-35.04672 ;-2.645624)$ \\
\hline $5-9$ & 0.9713 & $<0.001$ & -30.80917 & $(-34.54242 ;-27.07593)$ \\
\hline $10-14$ & - & - & - & $(-;-)$ \\
\hline 15-19 & 0.7647 & $<0.001$ & -23.2388 & $(-32.32247 ;-14.15513)$ \\
\hline $20-24$ & 0.8650 & $<0.001$ & -28.24621 & $(-36.1103 ;-20.38212)$ \\
\hline $25-29$ & 0.8314 & $<0.001$ & -25.00119 & $(-32.93499 ;-17.06738)$ \\
\hline $30-34$ & 0.8619 & $<0.001$ & -22.51058 & $(-28.85862 ;-16.16253)$ \\
\hline $35-39$ & 0.8620 & $<0.001$ & -18.72853 & $(-24.00947 ;-13.44759)$ \\
\hline $40-44$ & 0.7259 & $<0.001$ & -18.12083 & $(-25.96603 ;-10.27563)$ \\
\hline $45-49$ & 0.6504 & 0.002 & -13.35748 & $(-20.257 ;-6.457969)$ \\
\hline $50-54$ & 0.4089 & 0.025 & -8.606852 & $(-15.89853 ;-1.315178)$ \\
\hline $55-59$ & 0.1094 & 0.294 & -3.631847 & $(-10.93369 ; 3.669995)$ \\
\hline $60-64$ & 0.0149 & 0.706 & 1.09651 & $(-5.188218 ; 7.381239)$ \\
\hline $65-69$ & 0.0382 & 0.542 & 1.496552 & $(-3.791265 ; 6.784368)$ \\
\hline $70-74$ & 0.0750 & 0.389 & 2.21944 & $(-3.272787 ; 7.711668)$ \\
\hline
\end{tabular}


Table 1 (Continued)

\begin{tabular}{|c|c|c|c|c|}
\hline Age group (years old) & $\mathrm{R}_{\text {squad }}$ ** $^{2}$ & $P^{*}$ & $\beta$ & $\mathrm{Cl} 95$ \\
\hline $75-79$ & 0.5138 & 0.009 & 4.482028 & $(1.409971 ; 7.554085)$ \\
\hline$\geq 80$ & 0.5468 & 0.006 & 5.065513 & $(1.816483 ; 8.314543)$ \\
\hline Total & 0.7461 & $<0.001$ & -2.220774 & $(-3.133521 ;-1.308027)$ \\
\hline \multicolumn{5}{|l|}{ Sergipe } \\
\hline $0-4$ & 0.3958 & 0.028 & 16.80084 & (2.175946; 31.42573) \\
\hline $5-9$ & 0.0585 & 0.449 & 3.170491 & $(-5.795267 ; 12.13625)$ \\
\hline $10-14$ & 0.0081 & 0.782 & -1.360546 & $(-12.00007 ; 9.278984)$ \\
\hline $15-19$ & 0.1325 & 0.245 & 9.234521 & $(-7.415501 ; 25.88454)$ \\
\hline $20-24$ & 0.3218 & 0.054 & 22.27177 & $(-0.5121089 ; 45.05565)$ \\
\hline $25-29$ & 0.0294 & 0.594 & 6.73735 & $(-20.53732 ; 34.01202)$ \\
\hline $30-34$ & 0.1664 & 0.188 & 15.60095 & $(-9.000182 ; 40.20208)$ \\
\hline $35-39$ & 0.1184 & 0.274 & 9.745295 & $(-8.994871 ; 28.48546)$ \\
\hline $40-44$ & 0.1960 & 0.149 & 11.43506 & $(-4.882783 ; 27.75291)$ \\
\hline $45-49$ & 0.3376 & 0.048 & 18.72637 & $(0.2424035 ; 37.21034)$ \\
\hline $50-54$ & 0.1704 & 0.182 & 10.3418 & $(-5.738716 ; 26.42232)$ \\
\hline $55-59$ & 0.2305 & 0.114 & 12.73528 & $(-3.661189 ; 29.13174)$ \\
\hline $60-64$ & 0.3947 & 0.029 & 10.9791 & $(1.39947 ; 20.55874)$ \\
\hline $65-69$ & 0.1744 & 0.177 & 7.349407 & $(-3.91936 ; 18.61817)$ \\
\hline $70-74$ & 0.2338 & 0.111 & 11.01448 & $(-3.033541 ; 25.0625)$ \\
\hline $75-79$ & 0.0516 & 0.478 & 4.953654 & $(-10.01557 ; 19.92288)$ \\
\hline$\geq 80$ & 0.2689 & 0.084 & 6.095157 & $(-0.9869454 ; 13.17726)$ \\
\hline Total & 0.3880 & 0.031 & 1.75831 & $(0.2022819 ; 3.314338)$ \\
\hline \multicolumn{5}{|l|}{ Tocantins } \\
\hline $0-4$ & 0.0403 & 0.532 & 5.83771 & $(-14.24548 ; 25.9209)$ \\
\hline $5-9$ & 0.0395 & 0.536 & -3.49895 & $(-15.66093 ; 8.663035)$ \\
\hline $10-14$ & 0.2414 & 0.105 & -9.582572 & $(-21.55088 ; 2.385732)$ \\
\hline $15-19$ & 0.0188 & 0.671 & 4.52645 & $(-18.52391 ; 27.57681)$ \\
\hline $20-24$ & 0.1669 & 0.187 & -17.95299 & $(-46.21285 ; 10.30686)$ \\
\hline $25-29$ & 0.5149 & 0.009 & -40.35708 & $(-67.95664 ;-12.75752)$ \\
\hline $30-34$ & 0.4300 & 0.021 & -26.68918 & $(-48.34112 ;-5.037246)$ \\
\hline $35-39$ & 0.0059 & 0.812 & -3.062352 & $(-30.95675 ; 24.83204)$ \\
\hline $40-44$ & 0.2851 & 0.074 & 13.61374 & $(-1.576256 ; 28.80373)$ \\
\hline $45-49$ & 0.2540 & 0.095 & -7.518968 & $(-16.5988 ; 1.560868)$ \\
\hline $50-54$ & 0.0055 & 0.819 & -2.246457 & $(-23.50182 ; 19.00891)$ \\
\hline $55-59$ & 0.0336 & 0.568 & 5.983982 & $(-16.61707 ; 28.58503)$ \\
\hline $60-64$ & 0.4268 & 0.021 & -16.98793 & $(-30.8603 ;-3.115563)$ \\
\hline $65-69$ & 0.2260 & 0.118 & -9.908295 & $(-22.82922 ; 3.01263)$ \\
\hline $70-74$ & 0.0749 & 0.389 & 12.11104 & $(-17.88476 ; 42.10684)$ \\
\hline $75-79$ & 0.0138 & 0.716 & -3.402011 & $(-23.67377 ; 16.86975)$ \\
\hline$\geq 80$ & 0.1335 & 0.243 & 9.553773 & $(-7.597712 ; 26.70526)$ \\
\hline Total & 0.1780 & 0.172 & -0.895801 & $(-2.252156 ; 0.4605544)$ \\
\hline
\end{tabular}


318 Brazilian Incidence of TBI Takahashi et al.

Table 2 Analysis of the age-adjusted incidence of TBI in the female population in each state of Brazil (2008-2019)

\begin{tabular}{|c|c|c|c|c|}
\hline Age group (years) & R squad** & $P^{*}$ & $\beta$ & $\mathrm{Cl} 95$ \\
\hline \multicolumn{5}{|l|}{$\overline{S z s z}$} \\
\hline $0-4$ & - & - & - & $(-;-)$ \\
\hline $5-9$ & 0.4899 & 0.011 & -9.640286 & $(-16.57082 ;-2.709748)$ \\
\hline $10-14$ & 0.6157 & 0.003 & -7.263633 & $(-11.30695 ;-3.220314)$ \\
\hline $15-19$ & 0.2628 & 0.088 & -9.410898 & $(-20.51674 ; 1.694946)$ \\
\hline $20-24$ & 0.1755 & 0.175 & -7.70003 & $(-19.45788 ; 4.057818)$ \\
\hline $25-29$ & 0.0236 & 0.633 & -1.663256 & $(-9.199069 ; 5.872557)$ \\
\hline $30-34$ & 0.3267 & 0.052 & -6.539606 & $(-13.15462 ; 0.075404)$ \\
\hline $35-39$ & 0.5181 & 0.008 & -7.450187 & $(-12.51289 ;-2.387485)$ \\
\hline $40-44$ & 0.0032 & 0.862 & -.5330526 & $(-7.208636 ; 6.142531)$ \\
\hline $45-49$ & 0.0004 & 0.951 & -.2849064 & $(-10.3524 ; 9.782588)$ \\
\hline $50-54$ & 0.3524 & 0.042 & -10.44079 & $(-20.41326 ;-.4683075)$ \\
\hline 55 a 59 & 0.0911 & 0.340 & -5.438387 & $(-17.54201 ; 6.665235)$ \\
\hline $60-64$ & 0.1725 & 0.179 & -4.480862 & $(-11.39539 ; 2.433668)$ \\
\hline $65-69$ & 0.1608 & 0.196 & 4.175292 & $(-2.54566 ; 10.89624)$ \\
\hline $70-74$ & 0.0497 & 0.486 & -4.686002 & $(-19.11841 ; 9.746404)$ \\
\hline $75-79$ & 0.0034 & 0.857 & -.8548896 & $(-11.16229 ; 9.452509)$ \\
\hline$\geq 80$ & 0.3376 & 0.048 & -11.19307 & $(-22.23947 ;-.146666)$ \\
\hline Total & 0.4496 & 0.017 & -.8627143 & $(-1.535267 ;-.1901619)$ \\
\hline \multicolumn{5}{|l|}{ Alagoas } \\
\hline $0-4$ & 0.2165 & 0.127 & 3.507815 & $(-1.193722 ; 8.209352)$ \\
\hline $5-9$ & 0.0600 & 0.443 & -.7221193 & $(-2.735413 ; 1.291174)$ \\
\hline $10-14$ & 0.0311 & 0.584 & .7669556 & $(-2.24945 ; 3.783362)$ \\
\hline $15-19$ & 0.1811 & 0.168 & 3.633634 & $(-1.8115 ; 9.078767)$ \\
\hline $20-24$ & 0.1697 & 0.183 & 2.755433 & $(-1.538275 ; 7.049141)$ \\
\hline $25-29$ & 0.0541 & 0.467 & .9650897 & $(-1.879577 ; 3.809757)$ \\
\hline $30-34$ & 0.2329 & 0.112 & 2.295807 & $(-.6395447 ; 5.231158)$ \\
\hline $35-39$ & 0.0008 & 0.930 & -.1368907 & $(-3.532384 ; 3.258603)$ \\
\hline $40-44$ & 0.0060 & 0.810 & -.2019196 & $(-2.02733 ; 1.623491)$ \\
\hline $45-49$ & 0.0035 & 0.854 & .28787 & $(-3.112872 ; 3.688612)$ \\
\hline $50-54$ & 0.1531 & 0.208 & 1.948217 & $(-1.28022 ; 5.176654)$ \\
\hline $55-59$ & 0.0347 & 0.562 & .9667461 & $(-2.626933 ; 4.560425)$ \\
\hline $60-64$ & 0.0932 & 0.334 & 1.261954 & $(-1.510996 ; 4.034903)$ \\
\hline $65-69$ & 0.0054 & 0.821 & -.3569733 & $(-3.775757 ; 3.06181)$ \\
\hline $70-74$ & 0.0094 & 0.764 & .5403979 & $(-3.361631 ; 4.442427)$ \\
\hline $75-79$ & 0.0955 & 0.328 & 3.51014 & $(-4.099831 ; 11.12011)$ \\
\hline$\geq 80$ & 0.4304 & 0.021 & 2.913811 & $(.5519346 ; 5.275688)$ \\
\hline Total & 0.2368 & 0.109 & .2393597 & $(-.063446 ; .5421653)$ \\
\hline \multicolumn{5}{|l|}{ Amapá } \\
\hline $0-4$ & 0.0407 & 0.529 & 2.062617 & $(-4.99289 ; 9.118124)$ \\
\hline $5-9$ & 0.2022 & 0.142 & -4.555661 & $(-10.93155 ; 1.820232)$ \\
\hline $10-14$ & 0.5113 & 0.009 & -9.144175 & $(-15.44291 ;-2.845441)$ \\
\hline
\end{tabular}


Table 2 (Continued)

\begin{tabular}{|c|c|c|c|c|}
\hline Age group (years) & R squad** & $P^{*}$ & $\beta$ & $\mathrm{Cl} 95$ \\
\hline $15-19$ & 0.1218 & 0.266 & -10.28843 & $(-29.75654 ; 9.179667)$ \\
\hline $20-24$ & 0.1799 & 0.169 & -8.892631 & $(-22.27198 ; 4.486716)$ \\
\hline $25-29$ & 0.4149 & 0.024 & -13.67505 & $(-25.11652 ;-2.233582)$ \\
\hline $30-34$ & 0.0708 & 0.403 & -4.619878 & $(-16.41577 ; 7.176009)$ \\
\hline $35-39$ & 0.3997 & 0.027 & -23.6096 & $(-43.99557 ;-3.223641)$ \\
\hline $40-44$ & 0.5197 & 0.008 & -20.60429 & $(-34.56028 ;-6.648298)$ \\
\hline $45-49$ & 0.5033 & 0.010 & -32.27142 & $(-54.85898 ;-9.683858)$ \\
\hline $50-54$ & 0.4710 & 0.014 & -37.53286 & $(-65.55977 ;-9.505945)$ \\
\hline $55-59$ & 0.0655 & 0.422 & 3.451958 & $(-5.735439 ; 12.63935)$ \\
\hline $60-64$ & 0.6127 & 0.003 & -42.0611 & $(-65.62453 ;-18.49767)$ \\
\hline $65-69$ & 0.2410 & 0.105 & -14.3564 & $(-32.30588 ; 3.59308)$ \\
\hline $70-74$ & 0.4325 & 0.020 & -26.68285 & $(-48.22031 ;-5.145388)$ \\
\hline $75-79$ & 0.1354 & 0.239 & -11.21422 & $(-31.18402 ; 8.755573)$ \\
\hline$\geq 80$ & 0.0013 & 0.912 & -0.5636354 & $(-11.67384 ; 10.54657)$ \\
\hline Total & 0.5170 & 0.008 & -2.545576 & $(-4.279361 ;-0.8117918)$ \\
\hline \multicolumn{5}{|l|}{ Amazonas } \\
\hline $0-4$ & 0.5165 & 0.008 & 8.118629 & $(2.584474 ; 13.65278)$ \\
\hline $5-9$ & 0.0080 & 0.783 & .5615153 & $(-3.851824 ; 4.974855)$ \\
\hline $10-14$ & 0.1741 & 0.177 & 1.733952 & $(-.9267592 ; 4.394663)$ \\
\hline $15-19$ & 0.3032 & 0.064 & 5.030686 & $(-.3427634 ; 10.40414)$ \\
\hline $20-24$ & 0.0556 & 0.461 & 1.99757 & $(-3.802133 ; 7.797272)$ \\
\hline $25-29$ & 0.1418 & 0.228 & 2.67253 & $(-1.959724 ; 7.304785)$ \\
\hline $30-34$ & 0.0003 & 0.959 & -0.1129691 & $(-4.914523 ; 4.688585)$ \\
\hline $35-39$ & 0.2390 & 0.107 & 4.74971 & $(-1.221692 ; 10.72111)$ \\
\hline $40-44$ & 0.0294 & 0.594 & 1.172425 & $(-3.573821 ; 5.918672)$ \\
\hline $45-49$ & 0.0140 & 0.714 & 0.578578 & $(-2.836598 ; 3.993754)$ \\
\hline $50-54$ & 0.1874 & 0.160 & 5.252169 & $(-2.454004 ; 12.95834)$ \\
\hline $55-59$ & 0.1355 & 0.239 & 4.094851 & $(-3.193533 ; 11.38324)$ \\
\hline $60-64$ & 0.2115 & 0.133 & 6.748003 & $(-2.432457 ; 15.92846)$ \\
\hline $65-69$ & 0.3085 & 0.061 & 6.991178 & $(-.3834059 ; 14.36576)$ \\
\hline $70-74$ & 0.0436 & 0.515 & 2.230024 & $(-5.125422 ; 9.58547)$ \\
\hline $75-79$ & 0.3889 & 0.030 & 5.982722 & $(0.6989311 ; 11.26651)$ \\
\hline$\geq 80$ & 0.3798 & 0.033 & 8.995458 & $(0.8963964 ; 17.09452)$ \\
\hline Total & 0.4059 & 0.026 & 0.6679703 & $(0.0985558 ; 1.237385)$ \\
\hline \multicolumn{5}{|l|}{ Bahia } \\
\hline $0-4$ & - & - & - & $(-;-)$ \\
\hline $5-9$ & 0.2370 & 0.109 & -3.806058 & $(-8.618401 ; 1.006285)$ \\
\hline $10-14$ & 0.2134 & 0.131 & -2.222832 & $(-5.230155 ; .7844901)$ \\
\hline $15-19$ & 0.0155 & 0.700 & 1.055109 & $(-4.868402 ; 6.978619)$ \\
\hline $20-24$ & 0.0154 & 0.700 & 0.9724115 & $(-4.499277 ; 6.4441)$ \\
\hline $25-29$ & 0.0228 & 0.639 & 0.7382179 & $(-2.665531 ; 4.141967)$ \\
\hline $30-34$ & 0.0157 & 0.698 & 0.6846784 & $(-3.135397 ; 4.504754)$ \\
\hline $35-39$ & 0.0676 & 0.414 & 1.323999 & $(-2.140315 ; 4.788313)$ \\
\hline
\end{tabular}

(Continued) 
Table 2 (Continued)

\begin{tabular}{|c|c|c|c|c|}
\hline Age group (years) & R squad** & $P^{*}$ & $\beta$ & $\mathrm{Cl} 95$ \\
\hline $40-44$ & 0.1699 & 0.183 & 1.668083 & $(-0.9301996 ; 4.266366)$ \\
\hline $45-49$ & 0.1941 & 0.152 & 1.89844 & $(-0.8275053 ; 4.624386)$ \\
\hline $50-54$ & 0.5321 & 0.007 & 3.619254 & $(1.228115 ; 6.010393)$ \\
\hline $55-59$ & 0.0019 & 0.894 & 0.1439377 & $(-2.200638 ; 2.488513)$ \\
\hline $60-64$ & 0.1087 & 0.295 & 1.300524 & $(-1.323662 ; 3.92471)$ \\
\hline $65-69$ & 0.5568 & 0.005 & 4.071976 & $(1.512327 ; 6.631624)$ \\
\hline $70-74$ & 0.1823 & 0.166 & 2.078803 & $(-1.022925 ; 5.180531)$ \\
\hline $75-79$ & 0.7687 & $<0.001$ & 3.493241 & $(2.143242 ; 4.843241)$ \\
\hline$\geq 80$ & 0.7833 & $<0.001$ & 5.45145 & $(3.431327 ; 7.471574)$ \\
\hline Total & 0.1466 & 0.219 & 0.2581068 & $(-0.1806472 ; 0.6968607)$ \\
\hline \multicolumn{5}{|l|}{ Ceará } \\
\hline $0-4$ & 0.8561 & $<0.001$ & 31.22963 & $(22.20926 ; 40.25)$ \\
\hline $5-9$ & 0.0542 & 0.466 & 1.806035 & $(-3.508381 ; 7.120452)$ \\
\hline $10-14$ & 0.0142 & 0.713 & -.925012 & $(-6.364996 ; 4.514972)$ \\
\hline $15-19$ & 0.0057 & 0.815 & 1.272492 & $(-10.51981 ; 13.06479)$ \\
\hline $20-24$ & 0.0574 & 0.453 & -2.626295 & $(-10.12268 ; 4.870087)$ \\
\hline $25-29$ & 0.0116 & 0.739 & .8881604 & $(-4.892694 ; 6.669015)$ \\
\hline $30-34$ & 0.0280 & 0.603 & 1.429594 & $(-4.510157 ; 7.369345)$ \\
\hline $35-39$ & 0.1011 & 0.314 & 1.793781 & $(-1.975349 ; 5.562911)$ \\
\hline $40-44$ & 0.1717 & 0.180 & 3.284345 & $(-1.797733 ; 8.366423)$ \\
\hline $45-49$ & 0.0746 & 0.390 & 1.217114 & $(-1.803187 ; 4.237415)$ \\
\hline $50-54$ & 0.0006 & 0.937 & -0.1605315 & $(-4.60202 ; 4.280957)$ \\
\hline 55-59 & 0.2009 & 0.144 & 3.814583 & $(-1.54512 ; 9.174286)$ \\
\hline $60-64$ & 0.2078 & 0.136 & 2.319788 & $(-.8712304 ; 5.510807)$ \\
\hline $65-69$ & 0.0145 & 0.710 & 0.8608601 & $(-4.145333 ; 5.867053)$ \\
\hline $70-74$ & 0.5037 & 0.010 & 4.592762 & $(1.380598 ; 7.804925)$ \\
\hline $75-79$ & 0.4751 & 0.013 & 5.882353 & $(1.525782 ; 10.23892)$ \\
\hline$\geq 80$ & 0.6659 & 0.001 & 6.038874 & (3.025206; 9.052542) \\
\hline Total & 0.3441 & 0.045 & 0.6271852 & $(0.0170956 ; 1.237275)$ \\
\hline \multicolumn{5}{|l|}{ Distrito Federal } \\
\hline $0-4$ & 0.5719 & 0.004 & 48.87847 & (19.08292; 78.67402) \\
\hline $5-9$ & 0.2187 & 0.125 & 6.714877 & $(-2.226515 ; 15.65627)$ \\
\hline $10-14$ & 0.0003 & 0.956 & 0.1085961 & $(-4.158923 ; 4.376115)$ \\
\hline $15-19$ & 0.1623 & 0.194 & 4.129506 & $(-2.480444 ; 10.73945)$ \\
\hline $20-24$ & 0.0105 & 0.751 & 1.508598 & $(-8.81313 ; 11.83033)$ \\
\hline $25-29$ & 0.1298 & 0.250 & -2.556543 & $(-7.219703 ; 2.106617)$ \\
\hline $30-34$ & 0.0215 & 0.650 & -1.226807 & $(-7.065095 ; 4.611482)$ \\
\hline $35-39$ & 0.0001 & 0.980 & .0534951 & $(-4.536171 ; 4.643161)$ \\
\hline $40-44$ & 0.0546 & 0.465 & 2.351619 & $(-4.541663 ; 9.2449)$ \\
\hline $45-49$ & 0.0859 & 0.355 & 2.04306 & $(-2.652666 ; 6.738785)$ \\
\hline $50-54$ & 0.0743 & 0.391 & 2.217509 & $(-3.296344 ; 7.731361)$ \\
\hline $55-59$ & 0.3952 & 0.029 & 5.316369 & $(0.6819925 ; 9.950745)$ \\
\hline $60-64$ & 0.0288 & 0.598 & 1.385403 & $(-4.283945 ; 7.054751)$ \\
\hline
\end{tabular}


Table 2 (Continued)

\begin{tabular}{|c|c|c|c|c|}
\hline Age group (years) & R squad** & $P^{*}$ & $\beta$ & $\mathrm{Cl} 95$ \\
\hline $65-69$ & 0.0418 & 0.524 & 1.875528 & $(-4.448395 ; 8.199452)$ \\
\hline $70-74$ & 0.2565 & 0.093 & 6.793608 & $(-1.355956 ; 14.94317)$ \\
\hline $75-79$ & 0.2726 & 0.082 & 6.35147 & $(-.9593008 ; 13.66224)$ \\
\hline$\geq 80$ & 0.6430 & 0.002 & 9.530571 & $(4.526818 ; 14.53432)$ \\
\hline Total & 0.3818 & 0.032 & 0.9547533 & $(0.0986726 ; 1.810834)$ \\
\hline \multicolumn{5}{|l|}{ Espirito Santo } \\
\hline $0-4$ & 0.2126 & 0.131 & 9.350548 & $(-3.327267 ; 22.02836)$ \\
\hline $5-9$ & 0.3149 & 0.058 & -4.784494 & $(-9.757419 ; 0.1884304)$ \\
\hline $10-14$ & 0.3669 & 0.037 & -3.894091 & $(-7.49841 ;-.2897721)$ \\
\hline $15-19$ & 0.0025 & 0.877 & -.3142266 & $(-4.738197 ; 4.109743)$ \\
\hline $20-24$ & 0.0258 & 0.618 & -1.312679 & $(-6.993866 ; 4.368507)$ \\
\hline $25-29$ & 0.2139 & 0.130 & -4.787623 & $(-11.25495 ; 1.679702)$ \\
\hline $30-34$ & 0.1630 & 0.193 & -5.410094 & $(-14.04801 ; 3.227823)$ \\
\hline $35-39$ & 0.0230 & 0.638 & -0.9397241 & $(-5.257829 ; 3.378381)$ \\
\hline $40-44$ & 0.3581 & 0.040 & -5.41451 & $(-10.52224 ;-0.3067834)$ \\
\hline $45-49$ & 0.0971 & 0.324 & -2.494233 & $(-7.85185 ; 2.863383)$ \\
\hline $50-54$ & 0.0002 & 0.961 & 0.1265806 & $(-5.558383 ; 5.811545)$ \\
\hline $55-59$ & 0.0986 & 0.320 & 2.610574 & $(-2.950107 ; 8.171256)$ \\
\hline $60-64$ & 0.2222 & 0.122 & -4.080831 & $(-9.459912 ; 1.298251)$ \\
\hline $65-69$ & 0.0344 & 0.564 & 1.945182 & $(-5.314282 ; 9.204646)$ \\
\hline $70-74$ & 0.1145 & 0.282 & 3.57045 & $(-3.426145 ; 10.56704)$ \\
\hline $75-79$ & 0.1348 & 0.240 & 3.751684 & $(-2.945141 ; 10.44851)$ \\
\hline$\geq 80$ & 0.5358 & 0.007 & 7.846299 & $(2.700858 ; 12.99174)$ \\
\hline Total & 0.0023 & 0.882 & -0.0423119 & $(-0.662186 ; 0.5775622)$ \\
\hline \multicolumn{5}{|l|}{ Goiás } \\
\hline $0-4$ & 0.6813 & 0.001 & 11.74701 & $(6.086501 ; 17.40751)$ \\
\hline $5-9$ & 0.0461 & 0.503 & -1.282979 & $(-5.395496 ; 2.829538)$ \\
\hline $10-14$ & 0.7432 & $<0.001$ & -6.121299 & $(-8.656828 ;-3.585769)$ \\
\hline $15-19$ & 0.4217 & 0.022 & -4.675575 & $(-8.533874 ;-0.817275)$ \\
\hline $20-24$ & 0.2176 & 0.126 & -3.314525 & $(-7.743117 ; 1.114068)$ \\
\hline $25-29$ & 0.0846 & 0.359 & -1.736174 & $(-5.759694 ; 2.287347)$ \\
\hline $30-34$ & 0.4384 & 0.019 & -4.832585 & $(-8.686143 ;-0.9790279)$ \\
\hline $35-39$ & 0.3464 & 0.044 & -2.963998 & $(-5.83281 ;-0.0951861)$ \\
\hline $40-44$ & 0.0002 & 0.962 & 0.1107125 & $(-4.999881 ; 5.221306)$ \\
\hline $45-49$ & 0.1972 & 0.148 & -1.863849 & $(-4.513251 ; 0.7855516)$ \\
\hline $50-54$ & 0.0266 & 0.612 & 0.856575 & $(-2.792351 ; 4.505501)$ \\
\hline $55-59$ & 0.1384 & 0.234 & 1.504674 & $(-1.140637 ; 4.149986)$ \\
\hline $60-64$ & 0.2386 & 0.107 & 1.67024 & $(-0.4320956 ; 3.772575)$ \\
\hline $65-69$ & 0.2980 & 0.066 & 3.644382 & $(-0.2967489 ; 7.585512)$ \\
\hline $70-74$ & 0.4851 & 0.012 & 7.101298 & $(1.945838 ; 12.25676)$ \\
\hline $75-79$ & 0.6246 & 0.002 & 8.737753 & $(3.965034 ; 13.51047)$ \\
\hline$\geq 80$ & 0.6192 & 0.002 & 8.985107 & $(4.020176 ; 13.95004)$ \\
\hline Total & 0.1191 & 0.272 & 0.1756676 & $(-0.1609894 ; 0.5123246)$ \\
\hline
\end{tabular}

(Continued) 
322 Brazilian Incidence of TBI Takahashi et al.

Table 2 (Continued)

\begin{tabular}{|c|c|c|c|c|}
\hline Age group (years) & $\mathrm{R}_{\text {squad }}$ ** & $P^{*}$ & $\beta$ & $\mathrm{Cl} 95$ \\
\hline \multicolumn{5}{|l|}{ Maranhão } \\
\hline $0-4$ & 0.5017 & 0.010 & 10.37098 & $(3.08819 ; 17.65378)$ \\
\hline $5-9$ & 0.3241 & 0.053 & 4.689824 & $(-.0825611 ; 9.46221)$ \\
\hline $10-14$ & 0.5179 & 0.008 & 5.438709 & $(1.74145 ; 9.135967)$ \\
\hline $15-19$ & 0.6955 & 0.001 & 14.24277 & $(7.602113 ; 20.88343)$ \\
\hline $20-24$ & 0.5723 & 0.004 & 9.57752 & $(3.74374 ; 15.4113)$ \\
\hline $25-29$ & 0.3845 & 0.031 & 5.948034 & $(.6457083 ; 11.25036)$ \\
\hline $30-34$ & 0.7432 & $<0.001$ & 11.47592 & $(6.722478 ; 16.22936)$ \\
\hline $35-39$ & 0.7021 & 0.001 & 11.49936 & $(6.221312 ; 16.77742)$ \\
\hline $40-44$ & 0.6771 & 0.001 & 8.019792 & $(4.117273 ; 11.92231)$ \\
\hline $45-49$ & - & - & - & $(-;-)$ \\
\hline $50-54$ & 0.7073 & 0.001 & 8.524698 & $(4.660429 ; 12.38897)$ \\
\hline $55-59$ & 0.5089 & 0.009 & 7.817553 & $(2.406162 ; 13.22894)$ \\
\hline $60-64$ & 0.5195 & 0.008 & 8.023462 & (2.586399; 13.46053) \\
\hline $65-69$ & 0.8977 & $<0.001$ & 10.40719 & (7.932214; 12.88217) \\
\hline $70-74$ & 0.7468 & $<0.001$ & 9.892883 & $(5.834389 ; 13.95138)$ \\
\hline $75-79$ & 0.4902 & 0.011 & 11.06561 & $(3.113979 ; 19.01724)$ \\
\hline 80 & 0.7339 & $<0.001$ & 14.34985 & $(8.261023 ; 20.43867)$ \\
\hline Total & 0.9068 & $<0.001$ & 1.568764 & $(1.214362 ; 1.923167)$ \\
\hline \multicolumn{5}{|l|}{ Mato Grosso } \\
\hline $0-4$ & 0.3727 & 0.035 & 16.11461 & $(1.384131 ; 30.84508)$ \\
\hline $5-9$ & 0.2242 & 0.120 & -4.848429 & $(-11.20354 ; 1.506681)$ \\
\hline $10-14$ & 0.4076 & 0.025 & -10.74579 & $(-19.87406 ;-1.617525)$ \\
\hline $15-19$ & 0.4481 & 0.017 & -10.60045 & $(-18.88882 ;-2.312091)$ \\
\hline $20-24$ & 0.0071 & 0.795 & -1.092405 & $(-10.19218 ; 8.00737)$ \\
\hline $25-29$ & 0.3858 & 0.031 & -9.180187 & $(-17.34145 ;-1.018927)$ \\
\hline $30-34$ & - & - & - & $(-;-)$ \\
\hline $35-39$ & 0.2871 & 0.073 & -6.446691 & $(-13.60368 ; .7102961)$ \\
\hline $40-44$ & 0.0007 & 0.933 & -0.3095392 & $(-8.323214 ; 7.704135)$ \\
\hline $45-49$ & 0.0557 & 0.460 & -2.527613 & $(-9.857131 ; 4.801905)$ \\
\hline $50-54$ & 0.0206 & 0.657 & -1.203236 & $(-7.053678 ; 4.647207)$ \\
\hline $55-59$ & 0.2776 & 0.078 & -5.083865 & $(-10.86183 ; .6940999)$ \\
\hline $60-64$ & 0.2131 & 0.131 & -3.647757 & $(-8.586333 ; 1.290819)$ \\
\hline $65-69$ & 0.0005 & 0.945 & -0.1537923 & $(-5.009172 ; 4.701587)$ \\
\hline $70-74$ & 0.0005 & 0.942 & -0.1938531 & $(-6.031791 ; 5.644085)$ \\
\hline $75-79$ & 0.3308 & 0.050 & 4.016834 & $(-.008386 ; 8.042054)$ \\
\hline$\geq 80$ & 0.4822 & 0.012 & 5.768456 & $(1.556668 ; 9.980243)$ \\
\hline Total & 0.1429 & 0.226 & -0.3582794 & $(-.976588 ; .2600292)$ \\
\hline \multicolumn{5}{|l|}{ Mato Grosso do Sul } \\
\hline $0-4$ & 0.0429 & 0.518 & -4.764701 & $(-20.62671 ; 11.09731)$ \\
\hline $5-9$ & 0.0537 & 0.468 & -2.876659 & $(-11.38268 ; 5.629361)$ \\
\hline $10-14$ & 0.2093 & 0.135 & -5.683861 & $(-13.46722 ; 2.099497)$ \\
\hline $15-19$ & 0.4869 & 0.012 & -11.60097 & $(-19.99198 ;-3.209957)$ \\
\hline
\end{tabular}


Table 2 (Continued)

\begin{tabular}{|c|c|c|c|c|}
\hline Age group (years) & R squad** & $P^{*}$ & $\beta$ & $\mathrm{Cl} 95$ \\
\hline $20-24$ & 0.0903 & 0.342 & -5.60849 & $(-18.14757 ; 6.930589)$ \\
\hline $25-29$ & 0.3107 & 0.060 & -7.941243 & $(-16.2762 ; 0.3937146)$ \\
\hline $30-34$ & 0.0337 & 0.568 & -2.894659 & $(-13.82018 ; 8.030861)$ \\
\hline $35-39$ & 0.3951 & 0.029 & -5.067434 & $(-9.485543 ;-0.6493257)$ \\
\hline $40-44$ & 0.1462 & 0.220 & -3.336418 & $(-9.016733 ; 2.343898)$ \\
\hline $45-49$ & 0.0037 & 0.850 & 0.6069821 & $(-6.384124 ; 7.598089)$ \\
\hline $50-54$ & 0.0017 & 0.899 & -0.353155 & $(-6.372434 ; 5.666124)$ \\
\hline $55-59$ & 0.3323 & 0.050 & 4.526225 & $(0.0057693 ; 9.046681)$ \\
\hline $60-64$ & 0.0115 & 0.740 & -0.7571834 & $(-5.694233 ; 4.179866)$ \\
\hline $65-69$ & 0.0000 & 0.996 & 0.0152821 & $(-6.354052 ; 6.384617)$ \\
\hline $70-74$ & 0.0475 & 0.496 & 2.203911 & $(-4.749383 ; 9.157206)$ \\
\hline $75-79$ & 0.0038 & 0.849 & 0.6175715 & $(-6.415179 ; 7.650322)$ \\
\hline$\geq 80$ & 0.3829 & 0.032 & 5.007343 & (0.5286203; 9.486066) \\
\hline Total & 0.1423 & 0.227 & -0.3790746 & $(-1.034735 ; 0.2765856)$ \\
\hline \multicolumn{5}{|l|}{ Minas Gerais } \\
\hline $0-4$ & 0.4528 & 0.016 & 7.947021 & $(1.791886 ; 14.10216)$ \\
\hline $5-9$ & 0.1374 & 0.236 & -1.925525 & $(-5.324604 ; 1.473553)$ \\
\hline $10-14$ & 0.5833 & 0.004 & -3.957313 & $(-6.314069 ;-1.600558)$ \\
\hline $15-19$ & 0.0000 & 0.996 & .0056472 & $(-2.216268 ; 2.227563)$ \\
\hline $20-24$ & 0.0074 & 0.791 & .2135994 & $(-1.532338 ; 1.959536)$ \\
\hline $25-29$ & 0.1762 & 0.174 & -1.004814 & $(-2.535704 ; .5260765)$ \\
\hline $30-34$ & 0.0206 & 0.656 & -.5293417 & $(-3.101702 ; 2.043019)$ \\
\hline $35-39$ & 0.0024 & 0.880 & -.1668899 & $(-2.567324 ; 2.233544)$ \\
\hline $40-44$ & 0.0022 & 0.884 & -.1736082 & $(-2.753668 ; 2.406452)$ \\
\hline $45-49$ & 0.0189 & 0.670 & 0.4260096 & $(-1.736471 ; 2.58849)$ \\
\hline $50-54$ & 0.1149 & 0.281 & 1.045418 & $(-0.9990412 ; 3.089878)$ \\
\hline 55-59 & 0.1534 & 0.208 & 1.462447 & $(-0.9579588 ; 3.882853)$ \\
\hline $60-64$ & 0.5276 & 0.007 & 2.672733 & $(0.8907879 ; 4.454677)$ \\
\hline $65-69$ & 0.4303 & 0.021 & 2.966774 & (0.5613986; 5.372149) \\
\hline $70-74$ & - & - & - & $(-;-)$ \\
\hline $75-79$ & 0.6651 & 0.001 & 4.785735 & $(2.393157 ; 7.178313)$ \\
\hline$\geq 80$ & 0.9655 & $<0.001$ & 9.113131 & $(7.900097 ; 10.32617)$ \\
\hline Total & 0.4908 & 0.011 & 0.264662 & $(0.0747153 ; 0.4546088)$ \\
\hline \multicolumn{5}{|l|}{ Pará } \\
\hline $0-4$ & 0.6614 & 0.001 & 18.10461 & $(8.977609 ; 27.2316)$ \\
\hline $5-9$ & 0.4410 & 0.019 & 5.933646 & $(1.226817 ; 10.64047)$ \\
\hline $10-14$ & 0.1087 & 0.295 & 1.745147 & $(-1.776441 ; 5.266735)$ \\
\hline $15-19$ & 0.4457 & 0.018 & 10.02838 & $(2.147755 ; 17.909)$ \\
\hline $20-24$ & 0.6706 & 0.001 & 12.86556 & (6.511727; 19.21939) \\
\hline $25-29$ & 0.5837 & 0.004 & 7.83695 & (3.173694; 12.50021) \\
\hline $30-34$ & 0.3067 & 0.062 & 4.950075 & $(-.2935067 ; 10.19366)$ \\
\hline $35-39$ & 0.6336 & 0.002 & 9.088037 & $(4.219052 ; 13.95702)$ \\
\hline
\end{tabular}


324 Brazilian Incidence of TBI Takahashi et al.

Table 2 (Continued)

\begin{tabular}{|c|c|c|c|c|}
\hline Age group (years) & R squad s* $^{*}$ & $P^{*}$ & $\beta$ & $\mathrm{Cl} 95$ \\
\hline $40-44$ & 0.3176 & 0.056 & 6.329298 & $(-0.2083517 ; 12.86695)$ \\
\hline $45-49$ & 0.2869 & 0.073 & 3.392679 & $(-0.3756766 ; 7.161034)$ \\
\hline $50-54$ & 0.4334 & 0.020 & 4.639125 & $(0.9014347 ; 8.376816)$ \\
\hline $55-59$ & 0.0454 & 0.506 & 1.115735 & $(-2.487202 ; 4.718673)$ \\
\hline $60-64$ & - & - & - & $(-;-)$ \\
\hline $65-69$ & 0.5608 & 0.005 & 5.866574 & $(2.208725 ; 9.524424)$ \\
\hline $70-74$ & 0.2580 & 0.092 & 2.913897 & $(-0.5674948 ; 6.395289)$ \\
\hline $75-79$ & 0.2614 & 0.089 & 3.076177 & $(-0.5676788 ; 6.720032)$ \\
\hline$\geq 80$ & 0.4600 & 0.015 & 4.142049 & $(0.9796474 ; 7.304452)$ \\
\hline Total & 0.6473 & 0.002 & 1.041866 & (0.499994; 1.583738) \\
\hline \multicolumn{5}{|l|}{ Paraíba } \\
\hline $0-4$ & 0.3428 & 0.045 & -18.22192 & $(-36.00035 ;-.4434915)$ \\
\hline $5-9$ & 0.5617 & 0.005 & -14.51401 & $(-23.54803 ;-5.479985)$ \\
\hline $10-14$ & 0.6608 & 0.001 & -9.429877 & $(-14.18992 ;-4.669832)$ \\
\hline $15-19$ & 0.7566 & $<0.001$ & -25.20392 & $(-35.27776 ;-15.13008)$ \\
\hline $20-24$ & 0.6944 & 0.001 & -16.3593 & $(-24.00518 ;-8.713408)$ \\
\hline $25-29$ & 0.6740 & 0.001 & -14.44363 & $(-21.52075 ;-7.366516)$ \\
\hline $30-34$ & 0.7361 & $<0.001$ & -15.01083 & $(-21.34293 ;-8.678734)$ \\
\hline $35-39$ & 0.4425 & 0.018 & -11.06812 & $(-19.8219 ;-2.314332)$ \\
\hline $40-44$ & 0.4906 & 0.011 & -6.802076 & $(-11.68597 ;-1.918182)$ \\
\hline $45-49$ & 0.3687 & 0.036 & -4.199669 & $(-8.071472 ;-.3278662)$ \\
\hline $50-54$ & 0.5479 & 0.006 & -7.502873 & $(-12.30547 ;-2.700277)$ \\
\hline 55-59 & 0.2902 & 0.071 & -6.463661 & $(-13.58669 ; 0.6593715)$ \\
\hline $60-64$ & 0.5004 & 0.010 & -5.815274 & $(-9.909421 ;-1.721128)$ \\
\hline $65-69$ & 0.5442 & 0.006 & -5.808066 & $(-9.553055 ;-2.063078)$ \\
\hline $70-74$ & 0.3992 & 0.028 & -8.039083 & $(-14.98858 ;-1.089584)$ \\
\hline $75-79$ & 0.5322 & 0.007 & -7.892527 & $(-13.1059 ;-2.679154)$ \\
\hline$\geq 80$ & 0.3458 & 0.044 & -5.475623 & $(-10.78247 ;-.1687798)$ \\
\hline Total & 0.6593 & 0.001 & -1.822505 & $(-2.745552 ;-.8994569)$ \\
\hline \multicolumn{5}{|l|}{ Paraná } \\
\hline $0-4$ & 0.4633 & 0.015 & 25.90506 & $(6.258565 ; 45.55156)$ \\
\hline $5-9$ & 0.0063 & 0.807 & -1.220284 & $(-12.04901 ; 9.60844)$ \\
\hline $10-14$ & 0.3767 & 0.034 & -6.41554 & $(-12.23003 ;-0.6010457)$ \\
\hline $15-19$ & 0.0031 & 0.864 & -0.8178631 & $(-11.16461 ; 9.528888)$ \\
\hline $20-24$ & 0.1585 & 0.200 & 5.366183 & $(-3.346346 ; 14.07871)$ \\
\hline $25-29$ & 0.2206 & 0.123 & 6.264064 & $(-2.033255 ; 14.56138)$ \\
\hline $30-34$ & 0.0914 & 0.340 & 2.682787 & $(-3.276839 ; 8.642413)$ \\
\hline $35-39$ & 0.5297 & 0.007 & 4.775075 & $(1.605089 ; 7.945061)$ \\
\hline $40-44$ & 0.3054 & 0.062 & 5.900596 & $(-0.3696609 ; 12.17085)$ \\
\hline $45-49$ & 0.4876 & 0.012 & 6.102894 & $(1.695088 ; 10.5107)$ \\
\hline $50-54$ & 0.6135 & 0.003 & 7.046785 & $(3.105967 ; 10.9876)$ \\
\hline 55-59 & 0.3507 & 0.042 & 5.260538 & $(0.2170208 ; 10.30406)$ \\
\hline $60-64$ & 0.1668 & 0.188 & 3.24758 & $(-1.867253 ; 8.362412)$ \\
\hline
\end{tabular}


Table 2 (Continued)

\begin{tabular}{|c|c|c|c|c|}
\hline Age group (years) & R squad** & $P^{*}$ & $\beta$ & $\mathrm{Cl} 95$ \\
\hline $65-69$ & 0.8020 & $<0.001$ & 9.342576 & $(6.072279 ; 12.61287)$ \\
\hline $70-74$ & 0.7081 & 0.001 & 11.83228 & $(6.480057 ; 17.18449)$ \\
\hline $75-79$ & 0.7253 & $<0.001$ & 11.8577 & $(6.716543 ; 16.99885)$ \\
\hline 80 & 0.8689 & $<0.001$ & 14.17468 & $(10.29459 ; 18.05477)$ \\
\hline Total & 0.5165 & 0.008 & 1.113051 & $(.3542937 ; 1.871808)$ \\
\hline \multicolumn{5}{|l|}{ Pernambuco } \\
\hline $0-4$ & 0.4587 & 0.016 & 20.08197 & $(4.711813 ; 35.45213)$ \\
\hline $5-9$ & 0.1356 & 0.239 & -2.230095 & $(-6.1978 ; 1.73761)$ \\
\hline $10-14$ & 0.2002 & 0.145 & -2.807771 & $(-6.76172 ; 1.146178)$ \\
\hline $15-19$ & 0.0078 & 0.785 & -0.8289618 & $(-7.417808 ; 5.759884)$ \\
\hline $20-24$ & 0.0201 & 0.660 & 1.271014 & $(-4.976499 ; 7.518526)$ \\
\hline $25-29$ & 0.0286 & 0.599 & -1.40957 & $(-7.199286 ; 4.380146)$ \\
\hline $30-34$ & 0.0281 & 0.603 & -0.8938983 & $(-4.601126 ; 2.813329)$ \\
\hline $35-39$ & 0.0029 & 0.869 & -0.3303159 & $(-4.670242 ; 4.00961)$ \\
\hline $40-44$ & 0.0000 & 0.989 & -.0235981 & $(-3.877981 ; 3.830785)$ \\
\hline $45-49$ & 0.0102 & 0.755 & 0.3945688 & $(-2.340793 ; 3.129931)$ \\
\hline $50-54$ & 0.0349 & 0.561 & 1.09215 & $(-2.95232 ; 5.136619)$ \\
\hline $55-59$ & 0.5155 & 0.009 & 3.420278 & $(1.083794 ; 5.756763)$ \\
\hline $60-64$ & 0.0841 & 0.361 & 1.828415 & $(-2.423135 ; 6.079965)$ \\
\hline $65-69$ & 0.6943 & 0.001 & 4.835337 & $(2.574829 ; 7.095845)$ \\
\hline $70-74$ & 0.4248 & 0.022 & 5.095157 & $(0.9176525 ; 9.272662)$ \\
\hline $75-79$ & 0.4940 & 0.011 & 4.149871 & $(1.190355 ; 7.109386)$ \\
\hline$\geq 80$ & 0.7648 & $<0.001$ & 6.297119 & $(3.836662 ; 8.757576)$ \\
\hline Total & 0.1806 & 0.168 & 0.3994168 & $(-0.2000696 ; 0.9989032)$ \\
\hline \multicolumn{5}{|l|}{ Piauí } \\
\hline $0-4$ & 0.2228 & 0.121 & 2.721498 & $(-.8602594 ; 6.303255)$ \\
\hline $5-9$ & 0.0020 & 0.890 & .2298699 & $(-3.381449 ; 3.841189)$ \\
\hline $10-14$ & 0.0078 & 0.785 & -.764485 & $(-6.850879 ; 5.321909)$ \\
\hline $15-19$ & 0.0006 & 0.941 & .4824942 & $(-13.77715 ; 14.74214)$ \\
\hline $20-24$ & 0.1249 & 0.260 & 5.518982 & $(-4.772871 ; 15.81083)$ \\
\hline $25-29$ & 0.0727 & 0.397 & 3.959472 & $(-6.007446 ; 13.92639)$ \\
\hline $30-34$ & 0.4526 & 0.017 & 8.87165 & $(1.996943 ; 15.74636)$ \\
\hline $35-39$ & 0.7422 & $<0.001$ & 11.23719 & $(6.571171 ; 15.9032)$ \\
\hline $40-44$ & 0.3386 & 0.047 & 8.859709 & $(0.1344589 ; 17.58496)$ \\
\hline $45-49$ & 0.6257 & 0.002 & 8.866163 & $(4.034433 ; 13.69789)$ \\
\hline $50-54$ & 0.4652 & 0.015 & 7.177585 & $(1.755195 ; 12.59997)$ \\
\hline $55-59$ & 0.5469 & 0.006 & 5.05763 & $(1.814148 ; 8.301112)$ \\
\hline $60-64$ & 0.6501 & 0.002 & 8.002505 & $(3.865528 ; 12.13948)$ \\
\hline $65-69$ & 0.2702 & 0.083 & 6.217586 & $(-0.9823044 ; 13.41748)$ \\
\hline $70-74$ & 0.4629 & - & - & $(-;-)$ \\
\hline $75-79$ & - & 0.015 & 5.052122 & $(1.217552 ; 8.886692)$ \\
\hline$\geq 80$ & 0.5969 & 0.003 & 5.340481 & $(2.248443 ; 8.432518)$ \\
\hline Total & 0.6548 & 0.001 & 0.9228835 & $(0.4507541 ; 1.395013)$ \\
\hline
\end{tabular}

(Continued) 
Table 2 (Continued)

\begin{tabular}{|c|c|c|c|c|}
\hline Age group (years) & R squad s* $^{*}$ & $P^{*}$ & $\beta$ & Cl 95 \\
\hline \multicolumn{5}{|l|}{ Rio de Janeiro } \\
\hline $0-4$ & 0.6318 & 0.002 & 25.39181 & $(11.7352 ; 39.04842)$ \\
\hline $5-9$ & 0.0235 & 0.634 & 1.044656 & $(-3.699089 ; 5.788402)$ \\
\hline $10-14$ & 0.1143 & 0.282 & 1.274517 & $(-1.22543 ; 3.774465)$ \\
\hline $15-19$ & 0.3635 & 0.038 & 3.07803 & $(0.2080303 ; 5.94803)$ \\
\hline $20-24$ & 0.3429 & 0.045 & 3.044268 & $(0.0750017 ; 6.013534)$ \\
\hline $25-29$ & 0.4372 & 0.019 & 3.283671 & $(0.6587477 ; 5.908594)$ \\
\hline $30-34$ & 0.4502 & 0.017 & 3.546657 & $(0.7853276 ; 6.307986)$ \\
\hline $35-39$ & 0.6022 & 0.003 & 2.727076 & $(1.165361 ; 4.28879)$ \\
\hline $40-44$ & 0.7576 & $<0.001$ & 3.944793 & $(2.372393 ; 5.517194)$ \\
\hline $45-49$ & 0.4223 & 0.022 & 2.177646 & $(.3829085 ; 3.972383)$ \\
\hline $50-54$ & 0.6801 & 0.001 & 3.636395 & $(1.879112 ; 5.393679)$ \\
\hline $55-59$ & 0.6668 & 0.001 & 3.609583 & $(1.811849 ; 5.407318)$ \\
\hline $60-64$ & 0.8359 & $<0.001$ & 3.613389 & (2.485259; 4.741519) \\
\hline $65-69$ & 0.8193 & $<0.001$ & 3.776679 & $(2.52709 ; 5.026268)$ \\
\hline $70-74$ & 0.8414 & $<0.001$ & 6.629559 & $(4.601678 ; 8.657439)$ \\
\hline $75-79$ & 0.7519 & $<0.001$ & 5.432895 & $(3.234131 ; 7.63166)$ \\
\hline$\geq 80$ & 0.9472 & $<0.001$ & 6.980942 & $(5.81968 ; 8.142204)$ \\
\hline Total & 0.7483 & $<0.001$ & 0.8319257 & $(0.4920033 ; 1.171848)$ \\
\hline \multicolumn{5}{|c|}{ Rio Grande do Norte } \\
\hline $0-4$ & 0.6933 & 0.001 & 10.57031 & $(5.617228 ; 15.5234)$ \\
\hline $5-9$ & 0.1215 & 0.267 & -1.689138 & $(-4.889686 ; 1.51141)$ \\
\hline $10-14$ & 0.3000 & 0.065 & 2.557076 & $(-0.1952337 ; 5.309386)$ \\
\hline $15-19$ & 0.0164 & 0.692 & -0.7450692 & $(-4.811971 ; 3.321833)$ \\
\hline $20-24$ & 0.0021 & 0.887 & -0.2904265 & $(-4.747529 ; 4.166676)$ \\
\hline $25-29$ & 0.4388 & 0.019 & 5.454346 & $(1.107963 ; 9.800728)$ \\
\hline $30-34$ & 0.0187 & 0.672 & 0.8518794 & $(-3.497694 ; 5.201453)$ \\
\hline $35-39$ & 0.1257 & 0.258 & 2.526231 & $(-2.167559 ; 7.220022)$ \\
\hline $40-44$ & - & - & - & $(-;-)$ \\
\hline $45-49$ & 0.1494 & 0.215 & 1.848374 & $(-1.259065 ; 4.955814)$ \\
\hline $50-54$ & 0.2019 & 0.143 & 2.947943 & $(-1.181405 ; 7.077291)$ \\
\hline $55-59$ & 0.2493 & 0.098 & 3.207276 & $(-0.7140284 ; 7.128581)$ \\
\hline $60-64$ & 0.0402 & 0.532 & 1.209508 & $(-2.952159 ; 5.371175)$ \\
\hline $65-69$ & 0.1228 & 0.264 & 2.21825 & $(-1.958507 ; 6.395007)$ \\
\hline $70-74$ & 0.3081 & 0.061 & 3.545573 & $(-0.1981379 ; 7.289283)$ \\
\hline $75-79$ & 0.2432 & 0.103 & 2.211293 & $(-0.5371806 ; 4.959767)$ \\
\hline$\geq 80$ & 0.4372 & 0.019 & 3.840803 & $(0.7700599 ; 6.911545)$ \\
\hline Total & 0.4736 & 0.013 & 0.4305558 & $(0.1107277 ; 0.7503838)$ \\
\hline \multicolumn{5}{|l|}{ Rio Grande do Sul } \\
\hline $0-4$ & 0.6408 & 0.002 & -15.62972 & $(-23.8758 ;-7.383646)$ \\
\hline $5-9$ & 0.8498 & $<0.001$ & -16.04933 & $(-20.80282 ;-11.29584)$ \\
\hline $10-14$ & 0.8773 & $<0.001$ & -12.84923 & $(-16.23496 ;-9.463509)$ \\
\hline $15-19$ & 0.5200 & 0.008 & -10.64526 & $(-17.8522 ;-3.438316)$ \\
\hline
\end{tabular}


Table 2 (Continued)

\begin{tabular}{|c|c|c|c|c|}
\hline Age group (years) & R squad** & $P^{*}$ & $\beta$ & $\mathrm{Cl} 95$ \\
\hline $20-24$ & 0.8795 & $<0.001$ & -9.085543 & $(-11.45504 ;-6.716045)$ \\
\hline $25-29$ & 0.7025 & 0.001 & -7.737712 & $(-11.28574 ;-4.189688)$ \\
\hline $30-34$ & 0.6437 & 0.002 & -5.588676 & $(-8.51844 ;-2.658913)$ \\
\hline $35-39$ & 0.6304 & 0.002 & -5.775711 & $(-8.891471 ;-2.65995)$ \\
\hline $40-44$ & 0.4033 & 0.027 & -3.233923 & $(-6.005833 ;-.4620137)$ \\
\hline $45-49$ & 0.5362 & 0.007 & -4.088295 & $(-6.767621 ;-1.408969)$ \\
\hline $50-54$ & 0.6640 & 0.001 & -3.668585 & $(-5.507228 ;-1.829941)$ \\
\hline $55-59$ & 0.3108 & 0.060 & -2.873776 & $(-5.888748 ; .1411961)$ \\
\hline $60-64$ & 0.0133 & 0.721 & -0.2090245 & $(-1.477763 ; 1.059714)$ \\
\hline $65-69$ & 0.1683 & 0.185 & -1.66058 & $(-4.2615 ; .9403401)$ \\
\hline $70-74$ & 0.0345 & 0.563 & -0.483826 & $(-2.287273 ; 1.319621)$ \\
\hline $75-79$ & 0.0020 & 0.891 & .1759333 & $(-2.613862 ; 2.965728)$ \\
\hline$\geq 80$ & 0.6114 & 0.003 & 2.547342 & $(1.116432 ; 3.978252)$ \\
\hline Total & 0.7975 & $<0.001$ & -0.9685592 & $(-1.31249 ;-0.6246284)$ \\
\hline \multicolumn{5}{|l|}{ Rondônia } \\
\hline $0-4$ & 0.3065 & 0.062 & 28.20931 & $(-1.687353 ; 58.10597)$ \\
\hline $5-9$ & 0.0039 & 0.847 & -1.592208 & $(-19.48467 ; 16.30025)$ \\
\hline $10-14$ & 0.0007 & 0.937 & -0.4604056 & $(-13.17863 ; 12.25782)$ \\
\hline $15-19$ & 0.0689 & 0.410 & -5.530191 & $(-19.84996 ; 8.789579)$ \\
\hline $20-24$ & 0.1434 & 0.225 & -5.613616 & $(-15.27948 ; 4.052251)$ \\
\hline $25-29$ & 0.0003 & 0.960 & -.3173445 & $(-14.16972 ; 13.53503)$ \\
\hline $30-34$ & 0.2578 & 0.092 & -9.258983 & $(-20.32845 ; 1.810484)$ \\
\hline $35-39$ & 0.0037 & 0.851 & -1.411464 & $(-17.75854 ; 14.93561)$ \\
\hline $40-44$ & 0.0009 & 0.927 & -.6901954 & $(-17.07034 ; 15.68995)$ \\
\hline $45-49$ & 0.0003 & 0.960 & 0.1828823 & $(-7.719023 ; 8.084788)$ \\
\hline $50-54$ & 0.0001 & 0.975 & 0.1269122 & $(-8.52944 ; 8.783264)$ \\
\hline $55-59$ & 0.0076 & 0.788 & -1.758457 & $(-15.93503 ; 12.41812)$ \\
\hline $60-64$ & 0.0116 & 0.739 & -1.321833 & $(-9.925556 ; 7.28189)$ \\
\hline $65-69$ & 0.0801 & 0.373 & 3.339795 & $(-4.632843 ; 11.31243)$ \\
\hline $70-74$ & 0.0003 & 0.956 & -0.2438034 & $(-9.765434 ; 9.277827)$ \\
\hline $75-79$ & 0.0863 & 0.354 & 4.349799 & $(-5.622149 ; 14.32175)$ \\
\hline$\geq 80$ & 0.0072 & 0.794 & 1.106092 & $(-8.071634 ; 10.28382)$ \\
\hline Total & 0.0019 & 0.892 & 0.0911629 & $(-1.362938 ; 1.545264)$ \\
\hline \multicolumn{5}{|l|}{ Roraima } \\
\hline $0-4$ & 0.0318 & 0.579 & 12.59852 & $(-36.3679 ; 61.56495)$ \\
\hline $5-9$ & 0.1765 & 0.174 & -21.73233 & $(-54.81348 ; 11.34883)$ \\
\hline $10-14$ & 0.1959 & 0.150 & -7.627372 & $(-18.51471 ; 3.259968)$ \\
\hline $15-19$ & 0.0302 & 0.589 & 2.354127 & $(-7.03812 ; 11.74637)$ \\
\hline $20-24$ & 0.1593 & 0.199 & -7.963637 & $(-20.8541 ; 4.926824)$ \\
\hline $25-29$ & 0.0404 & 0.531 & -4.672425 & $(-20.72019 ; 11.37534)$ \\
\hline $30-34$ & 0.0628 & 0.432 & -5.990188 & $(-22.29562 ; 10.31524)$ \\
\hline $35-39$ & 0.0060 & 0.811 & 1.880183 & $(-15.14247 ; 18.90284)$ \\
\hline $40-44$ & 0.0028 & 0.870 & -1.073967 & $(-15.30852 ; 13.16058)$ \\
\hline
\end{tabular}

(Continued) 
Table 2 (Continued)

\begin{tabular}{|c|c|c|c|c|}
\hline Age group (years) & R squad ${ }^{* *}$ & $P^{*}$ & $\beta$ & Cl 95 \\
\hline $45-49$ & 0.0058 & 0.815 & 1.593803 & $(-13.16806 ; 16.35566)$ \\
\hline $50-54$ & 0.0049 & 0.829 & 1.350862 & $(-12.19512 ; 14.89684)$ \\
\hline $55-59$ & 0.0583 & 0.450 & 5.349706 & $(-9.805478 ; 20.50489)$ \\
\hline $60-64$ & 0.0163 & 0.692 & 2.459791 & $(-11.00169 ; 15.92128)$ \\
\hline $65-69$ & 0.1636 & 0.192 & 10.59231 & $(-6.279842 ; 27.46447)$ \\
\hline $70-74$ & 0.1079 & 0.297 & 10.04858 & $(-10.31028 ; 30.40744)$ \\
\hline $75-79$ & 0.1872 & 0.160 & 12.98364 & $(-6.078913 ; 32.0462)$ \\
\hline$\geq 80$ & 0.2349 & 0.110 & 18.71883 & $(-5.083237 ; 42.5209)$ \\
\hline Total & 0.0293 & 0.595 & 0.3087045 & $(-0.9438428 ; 1.561252)$ \\
\hline \multicolumn{5}{|l|}{ Santa Catarina } \\
\hline $0-4$ & 0.4635 & 0.015 & 12.65893 & $(3.063181 ; 22.25469)$ \\
\hline $5-9$ & 0.3392 & 0.047 & -8.596053 & $(-17.04961 ;-0.1424936)$ \\
\hline $10-14$ & 0.5455 & 0.006 & -9.097414 & $(-14.94826 ;-3.246571)$ \\
\hline $15-19$ & 0.5179 & 0.008 & -7.544919 & $(-12.67437 ;-2.415466)$ \\
\hline $20-24$ & 0.1140 & 0.283 & -2.681096 & $(-7.947777 ; 2.585585)$ \\
\hline $25-29$ & 0.4706 & 0.014 & -6.58917 & $(-11.51294 ;-1.665403)$ \\
\hline $30-34$ & 0.3523 & 0.042 & -3.423314 & $(-6.693576 ;-0.1530515)$ \\
\hline $35-39$ & 0.0000 & 1.000 & 0.0013251 & $(-4.842894 ; 4.845544)$ \\
\hline $40-44$ & - & - & - & $(-;-)$ \\
\hline $45-49$ & 0.1935 & 0.152 & -2.002779 & $(-4.884084 ; .8785263)$ \\
\hline $50-54$ & 0.0745 & 0.391 & 1.445936 & $(-2.144637 ; 5.03651)$ \\
\hline $55-59$ & 0.2682 & 0.085 & -3.431889 & $(-7.425865 ; 0.5620873)$ \\
\hline $60-64$ & 0.0299 & 0.591 & -0.9098262 & $(-4.560401 ; 2.740748)$ \\
\hline $65-69$ & 0.1565 & 0.203 & 2.267853 & $(-1.442422 ; 5.978128)$ \\
\hline $70-74$ & 0.2297 & 0.115 & 2.738095 & $(-.7951677 ; 6.271359)$ \\
\hline $75-79$ & 0.1945 & 0.151 & 1.88174 & $(-.8167319 ; 4.580211)$ \\
\hline$\geq 80$ & 0.6404 & 0.002 & 4.605765 & $(2.173735 ; 7.037796)$ \\
\hline Total & 0.1172 & 0.276 & -0.2215164 & $(-0.6499815 ; 0.2069486)$ \\
\hline \multicolumn{5}{|l|}{ São Paulo } \\
\hline $0-4$ & 0.2979 & 0.066 & -11.80236 & $(-24.5696 ; 0.9648859)$ \\
\hline $5-9$ & 0.7969 & $<0.001$ & -13.134 & $(-17.80647 ;-8.461533)$ \\
\hline $10-14$ & - & - & - & $(-;-)$ \\
\hline $15-19$ & 0.8327 & $<0.001$ & -8.932508 & $(-11.75412 ;-6.110899)$ \\
\hline $20-24$ & 0.6317 & 0.002 & -5.863624 & $(-9.018595 ;-2.708652)$ \\
\hline $25-29$ & 0.8142 & $<0.001$ & -5.255633 & $(-7.024561 ;-3.486705)$ \\
\hline $30-34$ & 0.8528 & $<0.001$ & -4.714968 & $(-6.09494 ;-3.334996)$ \\
\hline $35-39$ & 0.7227 & $<0.001$ & -3.524217 & $(-5.062283 ;-1.986151)$ \\
\hline $40-44$ & 0.7159 & 0.001 & -4.451312 & $(-6.427005 ;-2.475619)$ \\
\hline $45-49$ & 0.5225 & 0.008 & -3.060081 & $(-5.121413 ;-0.9987495)$ \\
\hline $50-54$ & 0.4941 & 0.011 & -2.921354 & $(-5.004346 ;-0.838363)$ \\
\hline $55-59$ & 0.3333 & 0.049 & -1.424822 & $(-2.844774 ;-0.0048694)$ \\
\hline $60-64$ & 0.4114 & 0.025 & -2.385287 & $(-4.395558 ;-0.3750159)$ \\
\hline $65-69$ & 0.4697 & 0.014 & -2.966908 & $(-5.188291 ;-0.7455247)$ \\
\hline
\end{tabular}


Table 2 (Continued)

\begin{tabular}{|c|c|c|c|c|}
\hline Age group (years) & R squad** & $P^{*}$ & $\beta$ & $\mathrm{Cl} 95$ \\
\hline $70-74$ & 0.3305 & 0.051 & -1.660837 & $(-3.326523 ; 0.0048494)$ \\
\hline $75-79$ & 0.1847 & 0.163 & -1.225717 & $(-3.040147 ; 0.5887135)$ \\
\hline$\geq 80$ & 0.0446 & 0.510 & 0.5650213 & $(-1.278493 ; 2.408535)$ \\
\hline Total & 0.7856 & $<0.001$ & -0.8349991 & $(-1.142387 ;-0.5276114)$ \\
\hline \multicolumn{5}{|l|}{ Sergipe } \\
\hline $0-4$ & 0.2919 & 0.070 & 7.947441 & $(-.7733108 ; 16.66819)$ \\
\hline $5-9$ & 0.0370 & 0.549 & 1.732965 & $(-4.498552 ; 7.964483)$ \\
\hline $10-14$ & 0.0214 & 0.650 & 0.8503887 & $(-3.20365 ; 4.904428)$ \\
\hline $15-19$ & 0.0108 & 0.748 & -0.8747333 & $(-6.766463 ; 5.016996)$ \\
\hline $20-24$ & 0.1134 & 0.284 & 2.246873 & $(-2.179173 ; 6.67292)$ \\
\hline $25-29$ & 0.0007 & 0.937 & -0.1429845 & $(-4.072993 ; 3.787024)$ \\
\hline $30-34$ & 0.0653 & 0.423 & 1.24914 & $(-2.081931 ; 4.580211)$ \\
\hline $35-39$ & 0.0179 & 0.678 & -0.8271689 & $(-5.141921 ; 3.487583)$ \\
\hline $40-44$ & 0.0230 & 0.638 & -1.12413 & $(-6.286454 ; 4.038194)$ \\
\hline $45-49$ & 0.3672 & 0.037 & -4.632597 & $(-8.917899 ;-0.3472946)$ \\
\hline $50-54$ & 0.0359 & 0.556 & 0.9140053 & $(-2.425486 ; 4.253497)$ \\
\hline $55-59$ & 0.0011 & 0.918 & -0.2288296 & $(-5.047579 ; 4.58992)$ \\
\hline $60-64$ & 0.0983 & 0.321 & -1.857997 & $(-5.821865 ; 2.105871)$ \\
\hline $65-69$ & 0.0182 & 0.676 & 0.7564922 & $(-3.162062 ; 4.675047)$ \\
\hline $70-74$ & 0.3549 & 0.041 & 4.111339 & $(0.2061061 ; 8.016572)$ \\
\hline $75--79$ & 0.0083 & 0.778 & 0.7755065 & $(-5.19098 ; 6.741993)$ \\
\hline$\geq 80$ & 0.0921 & 0.338 & 1.446853 & $(-1.753449 ; 4.647156)$ \\
\hline Total & 0.0714 & 0.401 & 0.1234257 & $(-0.1902398 ; 0.4370912)$ \\
\hline \multicolumn{5}{|l|}{ Tocantins } \\
\hline $0-4$ & 0.0034 & 0.858 & -1.215841 & $(-15.97209 ; 13.54041)$ \\
\hline $5-9$ & 0.0188 & 0.671 & -2.552168 & $(-15.54235 ; 10.43802)$ \\
\hline $10-14$ & 0.0028 & 0.871 & -0.4262147 & $(-6.128838 ; 5.276409)$ \\
\hline $15-19$ & 0.0528 & 0.473 & -4.152538 & $(-16.54989 ; 8.244811)$ \\
\hline $20-24$ & 0.0521 & 0.475 & -4.486744 & $(-17.96914 ; 8.995649)$ \\
\hline $25-29$ & 0.1499 & 0.214 & -5.246908 & $(-14.05069 ; 3.556878)$ \\
\hline $30-34$ & 0.0863 & 0.354 & -5.010362 & $(-16.49867 ; 6.477951)$ \\
\hline $35-39$ & 0.1541 & 0.207 & -6.409362 & $(-16.98886 ; 4.170133)$ \\
\hline $40-44$ & 0.2963 & 0.067 & -6.959401 & $(-14.51685 ; .5980507)$ \\
\hline $45-49$ & 0.0015 & 0.906 & -0.5955099 & $(-11.50913 ; 10.31811)$ \\
\hline $50-54$ & 0.0018 & 0.895 & -0.520531 & $(-9.048589 ; 8.007527)$ \\
\hline $55-59$ & 0.0357 & 0.557 & -1.48135 & $(-6.907307 ; 3.944608)$ \\
\hline $60-64$ & 0.0395 & 0.536 & -2.163091 & $(-9.678466 ; 5.352284)$ \\
\hline $65-69$ & 0.0331 & 0.571 & -3.530734 & $(-16.96922 ; 9.907754)$ \\
\hline $70-74$ & 0.0103 & 0.753 & 1.229576 & $(-7.243053 ; 9.702204)$ \\
\hline $75-79$ & 0.2603 & 0.090 & 6.372966 & $(-1.196761 ; 13.94269)$ \\
\hline$\geq 80$ & 0.1538 & 0.207 & -5.921126 & $(-15.70526 ; 3.863007)$ \\
\hline Total & 0.1291 & 0.251 & -0.4306934 & $(-1.219056 ; 0.3576693)$ \\
\hline
\end{tabular}

${ }^{*} p$ : linear regression.

${ }^{* *} r$ squad $\left(r^{2}\right)$ : predictive ability of the study. 
However, in Paraíba, all age ranges from both genders presented reduction in the admissions. The global reduction was of approximately twice among women and 7 times among men ( $\beta$ coefficient: -1.8 and -7.2 , respectively).

In Pernambuco, in the pediatric age range, there was a reduction among the boys in the 10 to14 age group ( $\beta$ coefficient: -10.5$)$, and a relevant increase among the girls in the 0 to 4 age group ( $\beta$ coefficient: 20.08). Among adults, the ages $>55$ years old presented an increase in the incidence in general, being more pronounced in the male gender.

In Piauí, there was a significant increase in admissions, present in all age ranges $>20$ years old in men and 30 years old in women. The group of men in the 20 to 24 age range presented the highest increase in the incidence of hospital admissions due to TBI in the country, with a $\beta$ coefficient of 63.43 .

Children from 0 to 4 years old were one of the most affected groups in Rio Grande do Norte ( $\beta$ coefficient: 11.86 and 10.57, boys and girls, respectively). Men, as a whole, presented a slight increase in the incidence ( $\beta$ coefficient: 1.63), more expressive from 50 to 54 years old ( $\beta$ coefficient: $20.65)$. In women, the variation was less significant, presenting only a small increase in the total incidence ( $\beta$ coefficient: 0.43).

In Sergipe, a reduction in the incidence of admissions was seen among women in the 45 to 49 age group ( $\beta$ coefficient: 4.63); however, among men in the same age range, there was an important increase ( $\beta$ coefficient: 18.72). In general, only the men obtained a statistically relevant increase in the incidence ( $\beta$ coefficient: 1.75).

\section{The Midwest Region (Goiás, Mato Grosso, Mato Grosso do Sul, and Distrito Federal)}

In Distrito Federal, both male and female genders presented an increase in the number of admissions in the age groups between 0 to 4 years old ( $\beta$ coefficient: 48.28 and 48.87 , respectively) and $\geq 80$ years old ( $\beta$ coefficient: 17.91 and 9.53 , respectively). In women, it was also observed an increase in the global incidence, considering all age groups ( $\beta$ coefficient: 0.95 ).

In Goiás, there was an increase in the incidence among males in the 10 to 29 ( $\beta$ coefficient: $10.02 ; 18.00 ; 19.42$; 17.15 respectively) and $\geq 70$ age groups ( $\beta$ coefficient: 18.32 ; $19.59 ; 17.07$, respectively). In women, there was an increase in the group between 0 and 4 years old ( $\beta$ coefficient: 11.74) and $\geq 70$ years old ( $\beta$ coefficient: $7.10 ; 8.73 ; 8.98$, respectively); on the other hand, there was a decrease in the age ranges from 10 to 19 years old ( $\beta$ coefficient: $-6.12 ;-4.67$, respectively) and 30 to 39 years old ( $\beta$ coefficient: -4.83 ; 2.96, respectively).

In Mato Grosso, there was an increase in the number of admissions in men from 0 to 4 years old ( $\beta$ coefficient: 18.76 ) and 70 to 74 years old ( $\beta$ coefficient: 20.48 ), although there was a decrease in the range from 5 to 9 years old ( $\beta$ coefficient: -12.65). In women, there was also an increase in the ranges from 0 to 4 years old ( $\beta$ coefficient: 16.11 ) and $\geq$ 75 years old ( $\beta$ coefficient: 4.01 and 5.76 , respectively), though there was a decrease in the age ranges from 10 to
19 years old ( $\beta$ coefficient: -10.74 and 10.60 , respectively) and 25 to 29 years old ( $\beta$ coefficient: -9.18 ).

In Mato Grosso do Sul, it was noticed a decrease among women in the following age ranges: 15 to 19 years old ( $\beta$ coefficient: - 11.60) and 35 to 44 years old ( $\beta$ coefficient: -5.06 and -3.33); on the other hand, there was an increase in the groups 55 to 59 years old ( $\beta$ coefficient: 4.52 ) and $\geq$ 80 years old (5.00). In men, it was noticed a decrease in the range from 5 to 9 years old ( $\beta$ coefficient: -16.37 ) and an increase in the ranges 70 to 74 years old ( $\beta$ coefficient: 17.94) and $\geq 80$ years old (19.00).

\section{The Southeast Region (São Paulo, Rio de Janeiro, Minas Gerais, and Espírito Santo)}

In Minas Gerais, there was an increase in the number of admissions among the male gender in the age ranges from 50 to 69 years old ( $\beta$ coefficient: $7.33 ; 12.63 ; 9.05 ; 11.15$, respectively) and $\geq 75$ years old ( $\beta$ coefficient: 11.06 and 14.06 , respectively); on the other hand, there was a decrease in the 5 to 14 age group ( $\beta$ coefficient: -6.87 and -13.43 , respectively). In the female gender, there was a decrease in the ranges from 10 to 14 years old ( $\beta$ coefficient: -3.95 ) and an increase in the ranges: 0 to 4 years old ( $\beta$ coefficient: 7.94$)$, 60 to 69 years old ( $\beta$ coefficient: 2.67 and 2.96 , respectively), 75 or more years old ( $\beta$ coefficient: 4.78 and 9.11 respectively) and in the total ( $\beta$ coefficient: 0.26 ).

In Espírito Santo, there was, in the male gender, a decrease in the ranges from 5 to 14 years old ( $\beta$ coefficient: -11.74 and - 13.10, respectively) and an increase in the ranges from 65 to 69 years old ( $\beta$ coefficient: 17.80) and 75 to more than 80 years old ( $\beta$ coefficient: 13.18 and 12.72 respectively). In women, there was a decrease in the ranges from 10 to 14 and 40 to 44 years old ( $\beta$ coefficient: -3.89 and -5.41 , respectively) and an increase in the range of 80 years old or more ( $\beta$ coefficient: 7.84).

In Rio de Janeiro, there was an increase in the number of admissions of men in all age ranges, except from 5 to 14 years old, including the total ( $\beta$ coefficient vary from 2.52 to 22.64 ). In women, there was an increase in all age ranges, except from 5 to 14 years old.

In São Paulo, there was a decrease in the incidence among men in the 0 to 54 age group and in the total ( $\beta$ coefficient: 2.22 ); on the other hand, there was an increase in the $\geq$ 75 years old group ( $\beta$ coefficient: 4.48 and 5.06, respectively). In women, there was a decrease in all age ranges, including the total, except in the 0 to 4 and 75 or more years old groups.

\section{The South Region (Paraná, Santa Catarina, and Rio Grande Do Sul)}

In Paraná, it was noticed an increase in the number of admissions among men in the groups 0 to 4 years old ( $\beta$ coefficient: 37.26 ) and 45 to more than 80 years old ( $\beta$ coefficient: $13.53,22.97,26.91,18.99,20.51,26.11,19.94$, 24.35 , respectively) and in the total ( $\beta$ coefficient: 1.74 ),; on the other hand, there was a decrease in the range from 10 to 14 years old ( $\beta$ coefficient: -16.30 ). In women, there was a decrease in the range from 10 to 14 years old $(\beta$ coefficient $-6.41)$ and an increase in the 0 to 4 years old $(\beta$ coefficient: 
$25.90), 35$ to 39 years old ( $\beta$ coefficient: 4.77 ), 45 to 59 years old ( $\beta$ coefficient: $9.34,11.83,11.85,14.17$, respectively), and 65 or more age groups ( $\beta$ coefficient: $9.34,11.83,11.85$, 14.17, respectively) as well as in the total ( $\beta$ coefficient: 1.11 ).

In Rio Grande do Sul, there was a decrease among men in the ranges from 0 to 54 years old ( $\beta$ coefficient: -22.05 , -29.96, -29.20, -26.69, - 34.53, -26.69, -34.53, -20,61, -19.39, $-14.88,-19.83,-17.85,-7.11$, respectively) and an increase in the ages of 70 or more years old ( $\beta$ coefficient: $6.75,5.22$ and 4.56 , respectively), besides the total decrease ( $\beta$ coefficient: -2.27). In women, there was a decrease in all age ranges except from 55 to 79 years old, including the total.

In Santa Catarina, in the male gender, there was a decrease in the age ranges from 10 to 24 years old ( $\beta$ coefficient: $14.32,-24.34$ and -24.87 , respectively) and from 45 to 49 years old ( $\beta$ coefficient: -8.85 ); on the other hand, there was an increase in the ranges from 70 to more than 80 years old ( $\beta$ coefficient: $3.85,13.79$ and 10.76 , respectively). In the female gender, there was an increase in the age range from 0 to 4 years old ( $\beta$ coefficient: 12.65 ) and 80 years old or more ( $\beta$ coefficient: 4.60 ) and a decrease in the ranges 5 to 19 years old ( $\beta$ coefficient: $-8.59,-9.09$ and -7.54 , respectively) and 25 to 34 years old ( $\beta$ coefficient: -6.58 and -3.42 , respectively).

\section{Discussion}

For an efficient analysis of the incidence of TBI as well as to correlate it to a possible growth or reduction in those incidence numbers, it is necessary to understand its different causes and their applicability in a thorough analysis, in which age groups play a fundamental part. Not only to differentiate the causes precisely, but also to propose preventive measures that are suitable to each respective cause in a more effective way, and thus prevent incapability and disability resulting of TBI. ${ }^{14}$ Besides preventing injury, an efficient public policy is mandatory for a great economy, since TBI generates immense expenses. ${ }^{15-19}$ Our study found significant data, which will be discussed in the following order:

1. Concerning the increase of TBI incidence in the Piaui and Maranhão region among young adults;

2. Increase of TBI incidence in the North, Northeast, South, and Midwest regions;

3. That increase was more pronounced on children (0-4 years old) and elderly ( $>65$ years old);

4. Paraíba has shown a significant decrease of TBI cases, which is a positive contrast in comparison with others Brazilian states.

In Brazil, the Southeast (42.5\%) and Northeast regions (25.8\%) dictate the major incidence of TBI, with more than a million cases of disability. ${ }^{1}$ The present paper's results indicate that the state of Piauí had the highest increase in incidence of TBI in the country in the last 10 years (coefficient $\beta=63.43$ e $p=0.002$ ). This increase in incidence was more pronounced amongst men in the 20 to 24 age range. The same age group among men had the highest increase in incidence in the state of Maranhão (coefficient $\beta=56.40$ e $p<0.001$ ). This can be explained by a series of events.
According to a 2017 edition of The Lancet, TBI is the major cause of mortality amongst young adults, and it is related to automobile accidents, violence, sports, and military conflict traumas. ${ }^{15}$ Therefore, the present study's results show a pressing need for more preventive campaigns, focusing on the education of young adults of the dangers of TBI, as well as ways to prevent it. The results also bring attention to how urgently the Brazilian preventive medicine stands in need of resources and incentive.

The main concern, though, is the increase in the TBI incidence amongst children ( $0-4$ years old) in the states of Ceará $(\beta=31.22$ and $p<0.001$ for boys; $\beta=42.20$ and $p<0.001$ for girls), Paraná $(\beta=37.26$ and $p=0.011$ for boys; $\beta=25.90$ and $p=0.015$ for girls), Pernambuco ( $\beta=20.08$ and $p=0.016$ for girls), Mato Grosso $(\beta=18.76$ and $p=0.005$ for boys; $\beta=16.11$ and $p=0.035$ for girls), and Distrito Federal $(\beta=48.87$ and $p=0.004$ for girls; $\beta=48.28$ and $p=0.006$ for boys). As for the older age groups, such as > 65 years old, a red light shows in the states of Roraima ( $\beta=31.04$ and $p=0.012$ for men older than 80 years old), Rondônia ( $\beta$ between 21.17-26.12 and $p<0.05$ for men older than 65 years old), Mato Grosso do Sul ( $\beta=19$ and $p<0.001$ for men older than 80 years old), and the Distrito Federal ( $\beta=17.91$ and $p=0.035$ for men older than 80 years old; $\beta=9.53$ and $p<0.002$ for women older than 80 years old). In developed countries, the highest incidences of TBI are amongst the age groups of 0 to 4,15 to 24 and $>65^{3}$ years old. Those numbers are compatible with the numbers found in Brazil, shown in this paper's analysis. A Brazilian study pointed out that among children under 10 years old, TBI's main external cause are falls (61.2\%), followed by transportation accidents (21.1\%). ${ }^{19}$ As for the elderly, the demographic transition had a big impact in the incidence increase in the country during the latest years; ${ }^{5,20}$ nevertheless, falls are still the leading cause.

In this context, it is important to highlight primary prevention as of great importance, since it is possible to bring awareness through educational campaigns to parents about the proper care with children during childhood, as well as campaigns aimed at the elderly and their caregivers of how to adequately prevent falls. A 30-year-experience study of a north American program of awareness called ThinkFirst was evaluated as very positive, since its implementation achieved significant reduction of the incidence in the pediatric population, as well as in adults and elderly. ${ }^{21}$ According to another study, after implementation of primary prevention programs in a medical reference center, a reduction of $22 \%$ of TBI amongst children was achieved. ${ }^{22}$ Therefore, programs such as this ought to be implemented in Brazil in order to mitigate the risks and, thus, diminish the national TBI incidence numbers.

Despite the alarming results in Northeast, the state of Paraíba stands out in a positive way. This was the only state in the Brazilian Northeast to show decreasing numbers in the incidence of TBI amongst men and women (coefficient $\beta=$ 18 and $p=0.004$ for men; coefficient $\beta=-7.2$ for women). Such results indicate an efficient and effective public health policy when it comes to TBI's precaution, and a functional 
notification system. The dissemination of these policies to other Brazilian states is important, and it can be implemented after any duly needed alterations based on each state's respective epidemiological profile.

\section{Conclusion}

The Brazilian Hospital Information System proves its fundamental importance, once it allows important studies such as the current one to be written, whose result's analysis might point out improvements that can be made. Besides that, it is remarkable the importance of redirecting public polices to preventive medicine since many of the TBI causes are avoidable through awareness and public health education of the population.

Place or Institution where the Work was Developed, City, and Country

Faculdade de Medicina do ABC, Santo André, SP, Brazil.

\section{Sources of Funding}

None.

\section{Ethics Approval and Consent to Participate}

The National Health Council of Brazil, by resolution $466 / 2012$, exempts this type of study from the research ethics committee, since it is a transversal study and all data is available on the internet free of charge and anonymously.

\section{Conflict of Interests}

The authors have no conflict of interests to declare.

\section{References}

1 Lira TML, Filho CRNL, Cavalcanti CAT, Lopes IMSS. As ciências biológicas nas dimensões humanista, crítica e reflexiva 2. Ponta Grossa: Editora Atena 2020. Available in https://www.atenaeditora.com.br/post-ebook/3151

2 Oliveira E, Lavrador JP, Santos MM, Lobo Antunes J. Traumatismo crânio-encefálico: abordagem integrada. Acta Med Port 2012;25 (03):179-192

3 Galgano M, Toshkezi G, Qiu X, Russell T, Chin L, Zhao LR. Traumatic Brain Injury: Current Treatment Strategies and Future Endeavors. Cell Transplant 2017;26(07):1118-1130

4 Dantas IEF, Oliveira TT, Neto CDM. Epidemiologia do traumatismo crânio encefálico (TCE) no nordeste no ano de 2012. REBES 2014;4 (01):18-23

5 Kleiven S, Peloso PM, von Holst H. The epidemiology of head injuries in Sweden from 1987 to 2000. Inj Control Saf Promot 2003;10(03):173-180
6 Winn HR, Bullock M, Hovda D, et al. Youmans Neurological Surgery. Epidemiol Traumat Brain Injury 2011;4(323): 3270-3275

7 Santos ME, De Sousa L, Castro-Caldas A. Epidemiologia dos traumatismos crânio-encefálicos em Portugal. Acta Med Port 2003;16(02):71-76

8 Tagliaferri F, Compagnone C, Korsic M, Servadei F, Kraus J. A systematic review of brain injury epidemiology in Europe. Acta Neurochir (Wien) 2006;148(03):255-268, discussion 268

9 Rutland-Brown W, Langlois JA, Thomas KE, Xi YL. Incidence of traumatic brain injury in the United States, 2003. J Head Trauma Rehabil 2006;21(06):544-548

10 Constâncio JF, et al. Perfil clínico-epidemiológico de indivíduos com histórico de traumatismo cranioencefálico. Rev Baiana Enferm 2018;32:1-10

11 Winn HR, Bullock M, Hovda D, et al. Youmans Neurological Surgery: Chapter 327-Neurochemical Pathomechanisms in Traumatic Brain injury. Elsevier Saunders; 201143305-3324

12 Eaton J, Hanif AB, Grudziak J, Charles A. Epidemiology, Management, and Functional Outcomes of Traumatic Brain Injury in SubSaharan Africa. World Neurosurg 2017;108:650-655

13 Ahmad OB, et al. GPE Discussion Paper Series: $N^{\circ} .31$ (ed World Health Organization) 1-14. 2001

14 Scholten AC, Haagsma JA, Panneman MJ, van Beeck EF, Polinder S. Traumatic brain injury in the Netherlands: incidence, costs and disability-adjusted life years. PLoS One 2014;9(10):e110905

15 Te Ao B, Brown P, Tobias M, et al; BIONIC Study Group. Cost of traumatic brain injury in New Zealand: evidence from a population-based study. Neurology 2014;83(18):1645-1652

16 Spitz G, McKenzie D, Attwood D, Ponsford JL. Cost prediction following traumatic brain injury: model development and validation. J Neurol Neurosurg Psychiatry 2016;87(02):173-180

17 Cassidy JD, Carroll LJ, Peloso PM, et al; WHO Collaborating Centre Task Force on Mild Traumatic Brain Injury. Incidence, risk factors and prevention of mild traumatic brain injury: results of the WHO Collaborating Centre Task Force on Mild Traumatic Brain Injury. J Rehabil Med 2004(43, Suppl):28-60

18 Maas AIR, Menon DK, Adelson PD, et al; InTBIR Participants and Investigators. Traumatic brain injury: integrated approaches to improve prevention, clinical care, and research. Lancet Neurol 2017;16(12):987-1048

19 Koizumi MS. Crianças Internadas por Traumatismo Crânio-Encefálico, no Brasil, 1998: Causas e Prevenção. Informe Epidemiol Brasília 2001

20 Brazinova A, Rehorcikova V, Taylor MS, et al. Epidemiology of traumatic brain injury in Europe: a living systematic review. J Neurotrauma 2016

21 Youngers EH, Zundel K, Gerhardstein D, et al. Comprehensive review of the ThinkFirst injury prevention programs: a 30-year success story for organized neurosurgery. Neurosurgery 2017;81 (03):416-421

22 Wehner DE, Sutton L. An interactive, hospital-based injury prevention program for first-, second-, and third-grade students. J Emerg Nurs 2005;31(04):383-386 